This manuscript is a preprint and will be shortly submitted for publication to a scientific journal. As a function of the peer-reviewing process that this manuscript will undergo, its structure and content may change.

If accepted, the final version of this manuscript will be available via the 'Peer-reviewed Publication DOI' link on the right-hand side of this webpage. Please feel free to contact any of the authors; we welcome feedback. 


\title{
Landslide susceptibility maps of Italy: lesson learnt from dealing with multiple landslide classes and the uneven spatial distribution of the national inventory
}

\author{
Marco Loche ${ }^{1}$, Massimiliano Alvioli ${ }^{2}$, Ivan Marchesini ${ }^{2}$, Haakon Bakka ${ }^{3}$, \\ and Luigi Lombardo ${ }^{4^{*}}$
}

${ }^{1}$ Institute of Hydrogeology, Engineering Geology and Applied Geophysics, Charles University, Albertov 6, 12843 Prague, Czech Republic

${ }^{2}$ Consiglio Nazionale delle Ricerche, Istituto di Ricerca per la Protezione Idrogeologica, via Madonna Alta 126, I-06128, Perugia, Italy

${ }^{3}$ Norwegian Veterinary Institute, Aas, Norway

${ }^{4}$ Faculty of Geo-Information Science and Earth Observation (ITC), University of Twente, PO Box 217, Enschede, AE 7500, The Netherlands

February 25, 2022

\begin{abstract}
Landslide susceptibility corresponds to the probability of landslide occurrence across a given geographic space. This probability is usually estimated by using a binary classifier which is informed of landslide presence/absence data and associated landscape characteristics. Here, we consider the Italian national landslide inventory to prepare slope-unit based landslide susceptibility maps. These maps are prepared for the eight types of mass movements existing in the inventory, (Complex, Deep Seated Gravitational Slope Deformation, Diffused Fall, Fall, Rapid Flow, Shallow, Slow Flow, Translational) and build one susceptibility map for each type. The analysis - carried out by using a Bayeian version of a Generalized Additive Model with a multiple intercept for each Italian region - revealed that the inventory may have been compiled with different levels of detail. This would be consistent with the datases being assembled from twenty sub-inventories, each prepared by different administrations of the Italian regions. As a result, this spatial inhonomegenity may lead to a biased national-scale susceptibility maps. On the basis of these considerations, we further analyzed the national database to confirm or reject the varying quality hypothesis suggested by the multiple intercepts results. For each landslide type, we then tried to build unbiased susceptibility models by removing regions with a poor landslide inventory from the calibration stage, and used them only as a prediction target of a simulation routine.
\end{abstract}


We analyzed the resulting eight maps finding out a congruent dominant pattern in the Alpine and Apennine sectors.

The whole procedure is implemented in R-INLA. This allowed to examine fixed (linear) and random (nonlinear) effects from an interpretative standpoint and produced a full prediction equipped with an estimated uncertainty.

We propose this overall modeling pipeline for any landslide datasets where a significant mapping bias may influence the susceptibility pattern over space.

Keywords: Integrated nested Laplace approximation (INLA), Landslide susceptibility, Slope unit, Model bias, Multiple landslide class 


\section{Introduction}

A landslide inventory is a catalog of the location of past landslides. It may contain a unique identification code for each landslide recorded and related information about type of landslide, state of activity, date of occurrence, material involved (Galli et al., 2008; Hervás and Bobrowsky, 2009). The inventory may be polygonal or point-based. And, it may correspond to an event-based inventory, in which all landslides have the same and simultaneous trigger, such as rainfall and earthquake (Iadanza et al., 2016; Cama et al., 2015; Fan et al., 2019; Loche et al., 2022). Or, it can encompass landslides with a ill-defined time of occurrence, which one would refer to as geomorhological inventory (Guzzetti et al., 2012).

National landslide inventories are geomorphological inventories in most cases. They may cover wide areas and, thus, may require different data (orthophotos or satellite images) and/or research groups to undertake the mapping effort. Unfortunately, when different data and/or groups are involved in the task, each output inventory inevitably suffers from the different quality and completeness (Guzzetti et al., 2012; Tanyaş and Lombardo, 2020; Pokharel et al., 2021) brought by some degree of subjectivity. For instance, some areas may be preferentially mapped, either for a specific choice, a topographic limitation, or for other reasons (Bornaetxea et al., 2018; Bornaetxea and Marchesini, 2021; Tanyas and Lombardo, 2020).

For example, Devoli et al. (2015) showed a significant presence of landslides around the Norwegian road network, for mapping at national scale is mostly undertaken by road authorities. The same preferential mapping was noted by Steger et al. (2021) in northern Italy or by Tanyaş et al. (2022) in eastern Turkey. Steger et al. (2016a) investigated bias effects due to specific land cover types, and Steger et al. (2016b) explored the same issue over a large portion of the Austrian territory, further extended to the whole Austria by Lima et al. (2017, 2021). Van Den Eeckhaut et al. (2012) and Kirschbaum et al. (2015) made similar considerations for the European and Global landslide catalogues, respectively. More recently, this topic has been also examined for the whole Chinese territory by Lin et al. (2021), who stressed the negative influence of an incomplete landslide inventory and the necessity to find ways to reduce the propagation of this spatial bias onto the final susceptibility map.

Similarly, the Italian national inventory was compiled by several groups, probably using different criteria. Trigila et al. (2010) discussed the quality of the Italian Landslide Inventory (known as IFFI, Trigila et al., 2007) and its completeness for individual administrative regions. However, few articles have used the IFFI information for susceptibility purposes. Iadanza et al. (2016) and Segoni et al. (2015) used it as a reference to extract rainfall triggering thresholds, whereas Bianchini et al. (2013) and Hölbling et al. (2012) used it to validate slope deformation detected through persistent scatterer interferometry. Colombo et al. (2005) adopted it to empirically study the hazard in the north-western Italian sector corresponding to the Piedmont region. Recently, Alvioli et al. (2021) adopted a subset of IFFI to partially validate simulations of rockfall trajectories with a three-dimensional model. Only one case exists where the authors considered the whole IFFI at the national 
scale (Marchesini et al., 2014), and only for validation, not for training a model.

Overall, the geomorphological literature lacks a unified/objective approach on how to deal with the propagation inventory biases to the resulting landslide susceptibility maps. The procedures presented in Steger et al. (2021) is currently the most comprehensive, and we will take inspiration from it in this work.

In terms of modeling approaches, the literature on landslide susceptibility features a large number of modeling techniques. The most common approach still belongs to the binomial Generalized Linear Model (GLM) or, as more specifically referred, to the Binary Logistic Regression (BLR) case, as also reported by Lombardo and Mai (2018) and Reichenbach et al. (2018). This method assumes that the distribution of landslide presences and absences across the geographic space can be explained according to a Bernoulli exponential distribution. And, that the influence of the covariates can be captured via linear relationships. This is usually implemented in a frequentist approach, tipically with good performances (e.g. Yesilnacar and Topal, 2005; Nefeslioglu et al., 2008; Rossi et al., 2010), which justifies the use of such a relatively simple model. Nevertheless, more complex statistical models are available nowadays, and they allow us to explore whether nonlinear relations between landslides and landscape characteristics exist. This is the case of the most common extension of the GLM framework, the Generalized Additive Model (GAM), already appeared in a number of applications (Goetz et al., 2011; Petschko et al., 2012; Goetz et al., 2021). However, even in such case, the frequentist framework does not allow to naturally account for uncertainties, which instead is naturally included in a Bayesian counterpart (Korup, 2021; Lombardo and Tanyas, 2021).

Few landslide susceptibility studies feature a Bayesian implementation. Das et al. (2012) show one example of Bayesian GLM to assess the landslide susceptibility in the proximity of roads in a Indian case study. Analogous examples can be found more recently in catchment (Lombardo et al., 2020; Luo et al., 2021) and and regional scale assessments (Tanyaş et al., 2021). Recently, Lombardo et al. $(2018 \mathrm{a}, 2019)$ proposed an extension of the Bayesian workflow pursued by the authors mentioned above by using a Log-Gaussian Point Process to predict landslide counts per mapping unit, this being implemented in R-INLA (Lindgren and Rue, 2015; Bakka et al., 2018).

Ultimately, another non-standardized approach in landslide science pertains to the way the space is partitioned i.e., which mapping unit is adopted. The vast majority of literature contributions opted for a regular mesh or grid-cell based subdivision (Sala et al., 2021; Arnone et al., 2016; Huang et al., 2017) whereas other researchers use Slope-Units (SU, Schlögel et al., 2018; Tanyas et al., 2019a,b; Alvioli et al., 2021). In very few cases, the differences induced by one or the other spatial partition are discussed (Erener and Düzgün, 2012; Alvioli et al., 2018; Ba et al., 2018; Jacobs et al., 2020; Doménech et al., 2020).

The grid cell-based partition type is regular, easy-to-use, and it usually subdivides the landscape at fine to very fine resolution. It is convenient because its resolution often coincides with satellite-derived data, but it leads to some operational issues. For instance, 
when a susceptible grid cell is surrounded by non-susceptible ones (Doménech et al., 2020), it is not straightforward to make decisions for landslide risk reduction nor for structural slope design. Conversely, SUs result from geomorphological processes which shape the landscape as much as the landslides, and have a physical correspondence on the terrain. Being medium-coarse in resolution, they require an aggregation step of the quantities one usually derives from satellite data. But, as they intrinsically express the morphodynamic behavior of a failing slope, SUs can be easily interpreted for master planning purposes. As a result of these advantages, although grid cells are still predominant in the literature, the number of SU-based applications has seen a constant increase in recent years, especially after automated and open access tools for SU delineation have been made available to the community (see, Alvioli et al., 2016). Considerations on the advantage of SU over grid-cells have been extensively discussed in Reichenbach et al. (2018).

In this work, we investigated landslide susceptibility in Italy considering the three aspects mentioned above: spatial homogeneity/heterogeneity of landslide inventories, a solid approach to the susceptibility classification, and use of SU as geomorphologically-sound mapping units. Specifically, we focus on examining possibly incomplete landslide inventories and develop a selection procedure to ensure that the bias they may generate would not propagate onto the final susceptibility maps. We do so within a GAM-type model built over a SU partition of the Italian territory. In doing so, we examine the (linear/nonlinear) covariate effects from which a suite of models that features an uncertainty estimation phase is also returned.

The present contribution is structured as follows: Section 2 provides the geographic context and a description of the National Landslide Inventory IFFI; Section 3 describes the statistical foundations; Sections 4 reports all the results, which are discussed in Section 5. Ultimately, Section 6 highlights strengths of the proposed workflow and suggests future improvements.

\section{Study Area}

The geomophology of Italy is unique and extremely diverse. Soldati and Marchetti (2017) prepared an outstanding compendium and overall description, where the national settings are dissected per region, geological history and anthropic influence.

Figure 1 summarizes the large scale geomorphological and geological setting of the country. The great variety of morphological forms is the result of an active geodynamic environment (Bosellini, 2017; Bartolini, 2010; Cowie et al., 2017), which determines a considerable variety in terms of outcropping lithologies (Bini, 2013). From a macroscopic, general and a naturalistic point of view, at least seven main geomorphological domains can be identified in Italy (Alps, Apennines, Po river alluvial valley, volcanoes, coasts, Sicily and Sardinia). This subdivision, however, is not able to depict the geomorphological differences that exist within these domains (Fredi and Lupia Palmieri, 2017). 


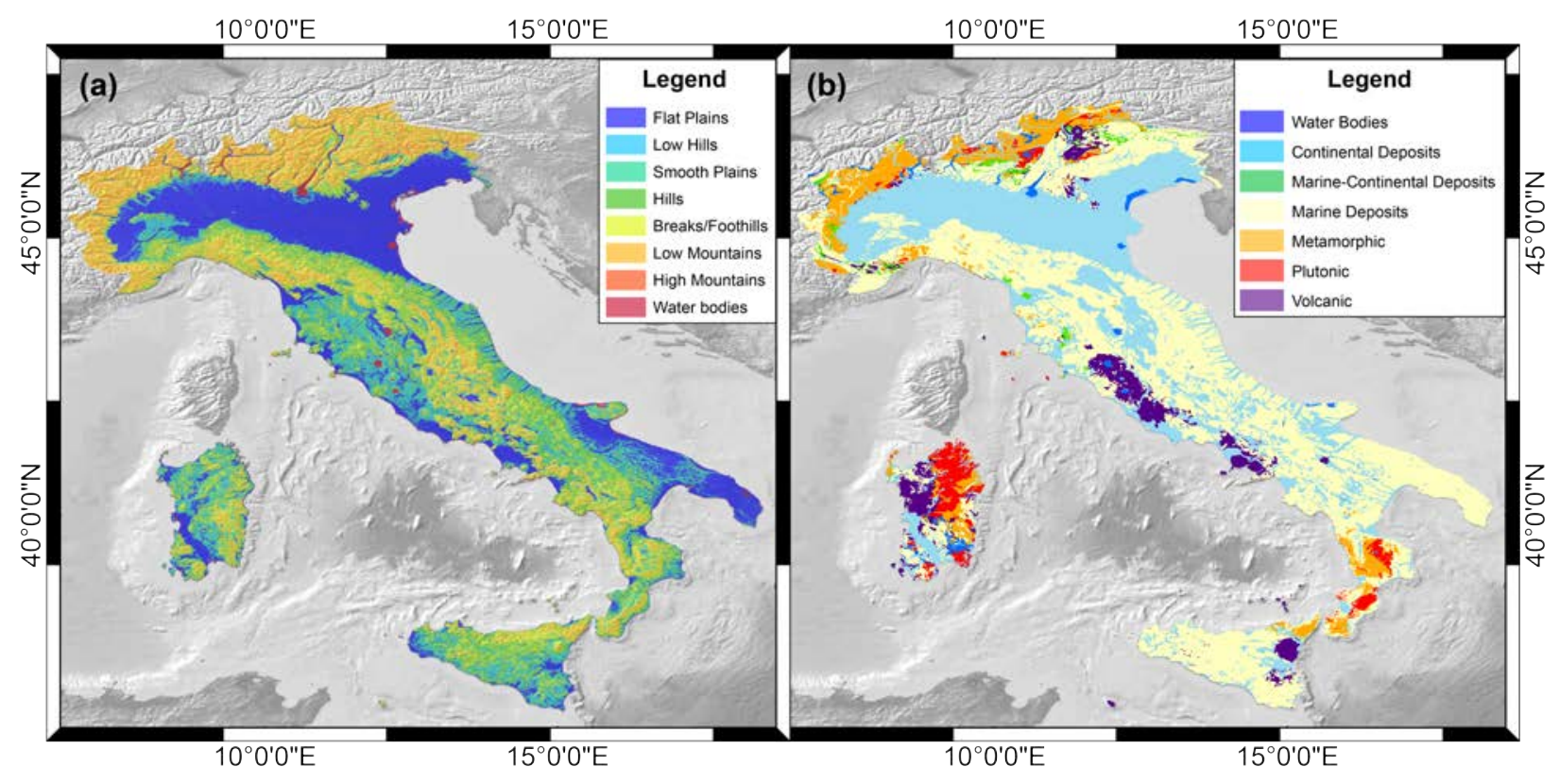

Figure 1: Geomorphological (a) and geological (b) settings of the study area. 
In a recent work, Alvioli et al. (2020) proposed a subdivision of the Italian territory into more than 300,000 Slope Units. In the same work, they analyzed the lithological and morphometric characteristics of 439 watersheds, of comparable size, covering the whole national territory and including the slope units. A clustering procedure allowed Alvioli et al. (2020) to define seven different land classes, characterized by different combinations of lithotypes and morphometries. These classes were found to correlate well with terrain elevation and other pre-existing morphological classifications of the territory (Guzzetti and Reichenbach, 1994; Drgu and Eisank, 2012). It is interesting to observe the spatial distribution of polygons belonging to the different seven classes (see Fig. 12 Alvioli et al., 2020). Although some of them are present mainly in specific geographical areas (e.g., the Alps), many others are widespread in different locations (from south to north and even on islands) and thus capture the geomorphological diversity mentioned by Fredi and Lupia Palmieri (2017).

Morphology and lithology are widely used in the literature to explain the spatial occurrence of landslides (Reichenbach et al., 2018). Consequently, in the remainder of this paper, we assumed that landslide information from the IFFI inventories should be quantitatively comparable within the same class although located in different regions of the country.

\subsection{Landslide inventory}

According to the IFFI catalogue (link here) landslides are non-uniformly distributed over Italy.

Figure 2 shows that mass movements are particularly dense in the Lombardia (LOM) region, and where the Alpine environment locally dominates the landscape. Moreover, a less dense but still large presence of landslides well aligns along the Appenine chain from the North to Central Italy, while landslide density appears to decrease in the South.

In the Apulia (PUG) region, this appears quite reasonable, for the landscape is relatively gentle. However, the IFFI inventory strikingly characterize Calabria (CAL) Sicilia (SIC) and Sardegna (SAR) as scarce in number of landslides. This may be already an indication of a uneven inventory. For example, in Sicily, IFFI reports 4,571 landslides out of which 48 are classified as rapid flows. Yet, several studies have reported for the same region a much larger number of superficial and fast mass movements. For instance, Bout et al. (2018); Van den Bout et al. (2021) modeled 395 debris flows only within the extremely small catchment of Itala, north-eastern SIC. Right next to Itala, Ardizzone et al. (2012) also mapped several hundreds of debris flows within the Briga and Giampilieri catchments. Similarly, Cama et al. (2017) mapped 810 debris flows in the small catchment of Saponara, on the other side of the Peloritan belt. More generally, Ciampalini et al. (2015) recognized diffused superficial deformations consistent with shallow landslides, over the whole Messina province. Thus, there maybe significant discrepancies between the information contained in the IFFI inventory and reality.

Despite local differences in terms of landslide distribution per region, the mapping criterion behind the IFFI record is to assign a landslide type to each mass movement. This 


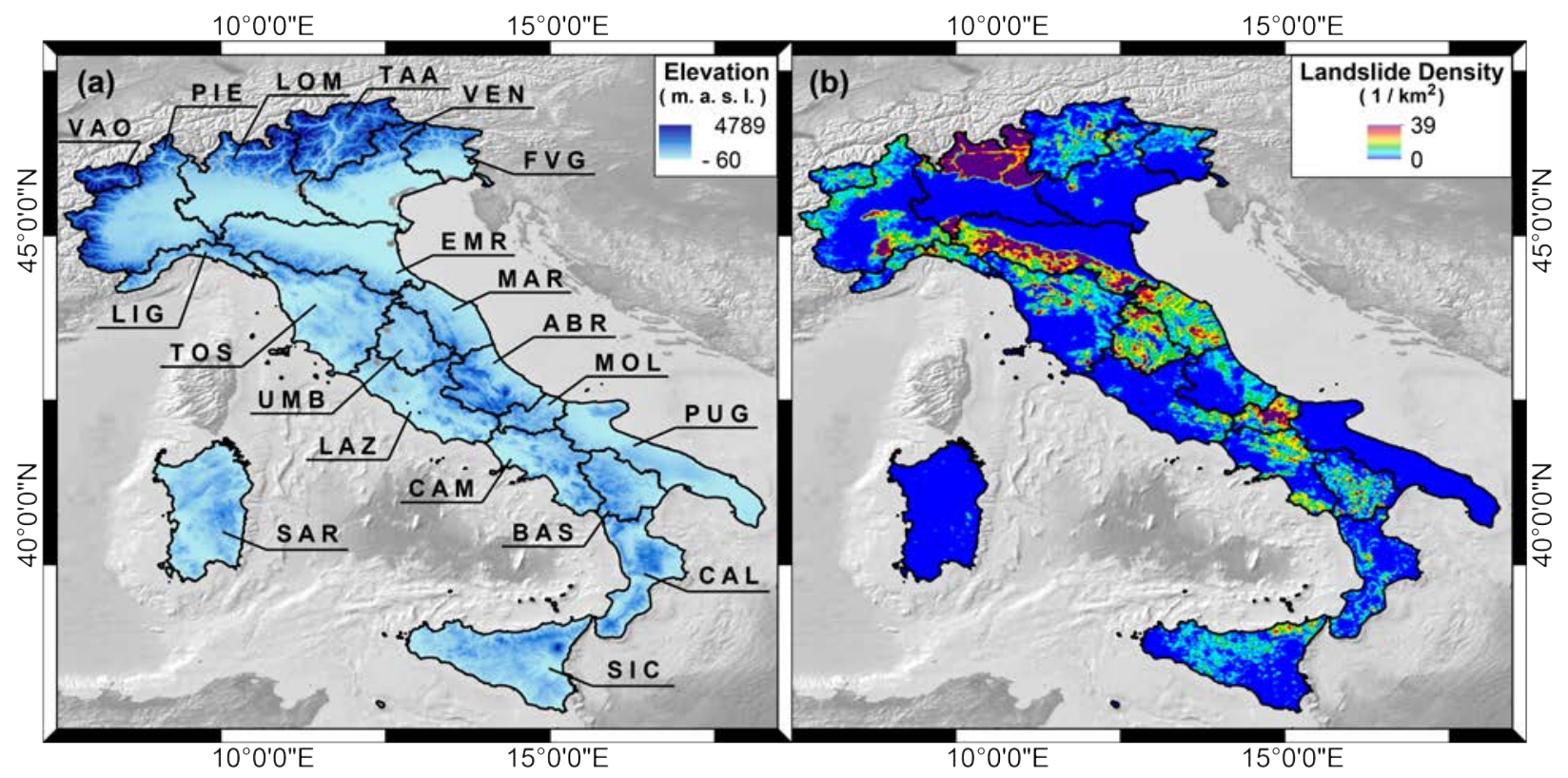

Figure 2: Administrative partition by region together with relative acronyms (a) and density map of the whole national landslide inventory (b). 
follows a non-standard geomorphological description of the failing mass by reporting the failing mechanism and the velocity of the moving mass (Hungr et al., 2014, sensu). This leads to eight classes summarized as follows:

1. Complex: this class includes landslides for which more than one failure mechanism was recognised. It corresponds to the Complex class described by Varnes (1978).

2. DSGSD: this class corresponds to deep-seated landslides described by Guerricchio et al. (2012).

3. Diffused Fall: this class does not strictly correspond to a single landslide type but combines Falls and Topples. Those who mapped the phenomena, could only recognise the talus without being able to discriminate the initiation mechanism. Thus, a "Diffused" class was created within the IFFI inventory to mark the two uncertain initiation processes.

4. Fall: this class corresponds to the Falls described in Varnes (1978).

5. Rapid Flow: this class encompasses flow-like mass movements, usually in unconsolidated materials and corresponds to the landslides characterized by a rapid to extremely rapid motion as reported in Hungr et al. (2014).

6. Shallow: this class consists of non-deep mass movements which are usually triggered by strong meteorological stresses which result in gravel/sand/debris slide activations as described in Hungr et al. (2014).

7. Slow Flow: this class encompasses mass movements with a slow motion usually involving clayey material. It corresponds to the dry (or non-liquefied) sand/silt/gravel/debris flow and lateral spreading types described in Hungr et al. (2014).

8. Translational: this class includes both the translational and rotational sliding as per Hungr et al. (2014).

Figure 3 shows a bar plot summarizing the regional distribution of the eight types of landslide classes listed above. The relative distribution of landslide types in different regions is very heterogeneous. Moreover, Figure 3 shows that in some regions certain landslide types are absent, or present in almost negligible quantities. One of the possible causes of this strong difference between regions can be linked to the physical characteristics of the territories. Certain types of landslides can only occur where given geomorphological conditions exist. However, among the causes of this heterogeneity, one may also consider the poor quality and completeness of the inventories, perhaps linked to deficiencies in terms of recognition, mapping and classification of landslides. 


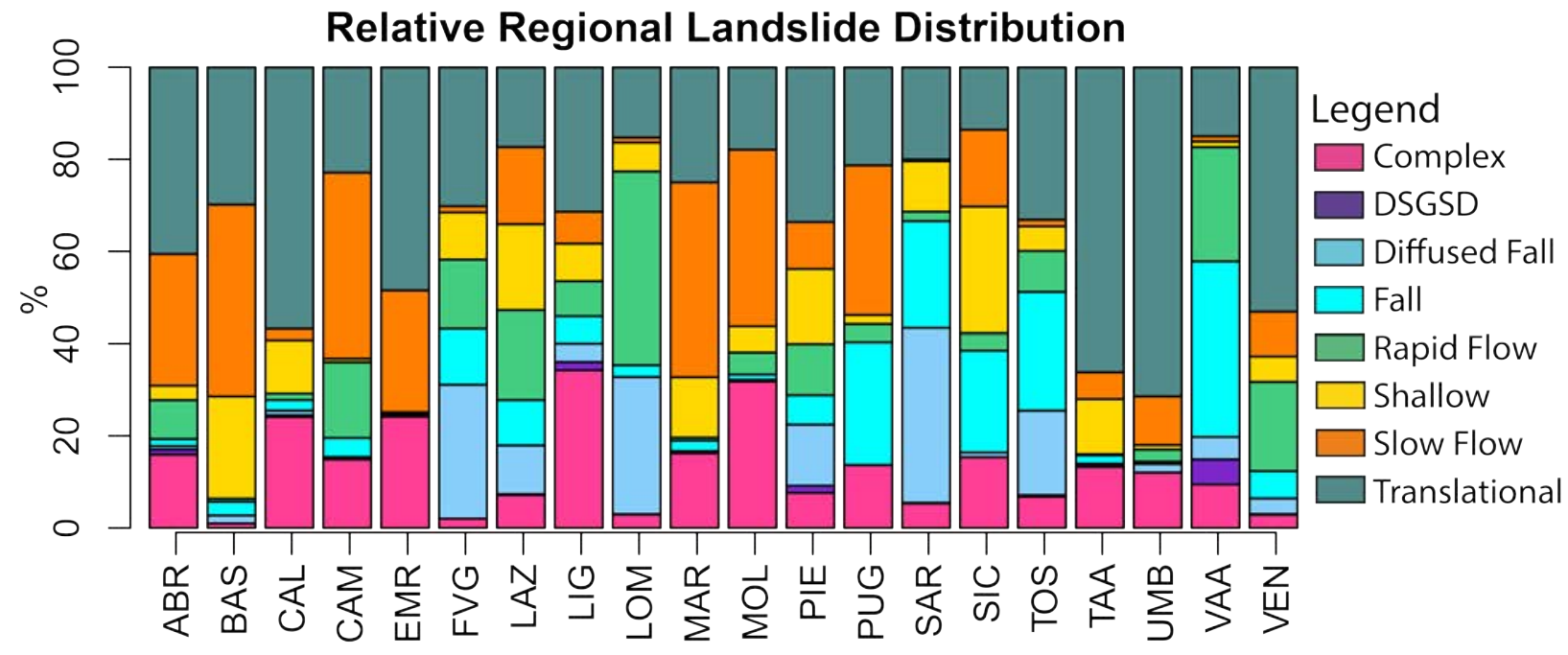

Figure 3: Stacked barplot of the landslide type distribution by region. The relative counts have been normalized per region and expressed in percentage. 


\subsection{Mapping Units}

The SU partition used in this work was first presented in Alvioli et al. (2020). There, the authors use the r.slopeunits software (Alvioli et al., 2016) to delineate SUs over the whole Italy. The SU dataset (link here) contains 325,578 slope unit polygons of varying shape and size. Each polygon is intended to encompass locally homogeneous terrain, from the aspect direction point of view, and thus it corresponds to a hillslope in the real world. The software used to delineate the polygons is adaptive, as it singles out SUs of different size in different geographical locations. Its input parameters are optimized using only elevation data. In particular, no landslide nor other terrain information enter the slope unit delineation procedure. This makes the SU map adopted in this work completely independent from the landslide inventory itself, and strongly related to the underlying topography, nation-wide.

We stress here that Alvioli et al. (2020) constrained SU delineation to remove flat or near-flat areas, obtaining a spatial partition associated with to landslides, i.e., slopes. This is also a criterion which has already appeared in other studies (e.g. Tanyaş et al., 2019a,b) to focus the predictive model on slopes where instabilities may be expected uniquely on the basis or topographic roughness and to limit the dataset in size to those areas which require attention.

The resulting SU cover $224,032 \mathrm{~km}^{2}$ out of the total $301,093 \mathrm{~km}^{2}$ of the Italian territory. This indication in itself stresses that $77 \%$ of the country is topographically rough and potentially prone to landslide just from a simple physiographic criterion.

Notably, combining the IFFI inventory and the SUs, each landslide class has a different number of SUs where at least one landslide fell into, which we report here: 26,960 Complex, 1,534 DSGSD, 14,960 Diffused Falls, 13,202 Falls, 16,478 Rapid Flows, 21,173 Shallow, 28,540 Slow Flows and 52,587 Translational landslides.

\subsection{Explanatory variables}

Due to the large size of the study area, and to the different types of landslides, we selected a large suite of explanatory variables (covariates hereafter) to support the model training phase. A sub-set of the covariate set corresponds to terrain characteristics reported in the landslide susceptibility studies (Budimir et al., 2015). To those, we added few more properties to describe the lithological and pedological signal across Italy, as well as the shape characteristics of the SU partition.

In Table 1 we list the whole set of covariates used to describe the landslide distribution across Italy. Notably, as also mentioned in Section 1, the use of SU requires an aggregation step to convert the distribution of covariates from grid cell level to SU level. We used mean and standard deviation - rarely this is also done by considering a quantile description of the covariates (Castro Camilo et al., 2017; Amato et al., 2019). We opted to use the mean and standard deviation assuming these two statistical moments to be sufficient in describing the covariate distribution per mapping unit (see Lombardo and Tanyas, 2020). We used all the 
covariates as linear effects, with the exception of few cases, which are reported in Table 1 , and for which we used non-linear effects; we provide an explanation on what this implies in Section 3.

Below we provide a further description on the covariates listed in Table 1. Geomorphologically, we included Slope, Aspect (in its continuous form through Eastness and Northness), Curvatures, Relative Slope Position and Topographic Wetness Index (TWI). These were computed from the $25 \mathrm{~m}$ DEM of Italy, EU-DEM, from Copernicus (link here).

Pedologically and, to some extent, lithologically, we considered soil attributes at $250 \mathrm{~m}$ resolution, obtained from Soilgrids global datasets (Hengl et al., 2017).

In addition, we believe that the shape of an SU itself may have an impact on landslide susceptibility, especially in this research, which aims at distinguishing several types of mass movements. To this end, we considered the Maximum Distance within an SU, calculated from the highest to the lowest point along an SU boundary. Similarly, we also computed a roundness/elongation index, computed as the Maximum Distance divided by the square root of the SU area. This index represents wide SUs when the ratio returns small values, and more and more elongated SUs as the ratio increases.

Ultimately, we initially used the administrative regions partitioning the country as an additional covariate, under the assumption that each region separately carries a potentially biasing signal due to the mapping procedure adopted among different administrations.

Further details on the actual implementation and covariates' use are provided in the following Section.

\section{Bayesian Generalized Additive Model}

\subsection{Bayesian models and inference with R-INLA}

We use Bayesian modeling, in the software R, with the R-package INLA (Rue et al., 2009).

Bayesian modelling means that we have a prior probability distribution on all parameters, and after we make observations, we get posterior probability distributions on these parameters. Specifying the priors is part of model building, and can either be done by giving priors that have very little information in them, as in this paper, or priors that are based on expert knowledge. To get a point estimate for a parameter, we find the mean of the posterior distribution, and to get the uncertainty, we find e.g. the $95 \%$ credible interval (CI), meaning an interval between the $2.5 \%$ quantile and the $97.5 \%$ quantile.

INLA is a popular tool for specifying and inferring Bayesian models, and is used in a wide range of relevant applications (Opitz et al., 2018; Pimont et al., 2021; Titti et al., 2021). INLA is short for Integrated Nested Laplace Approximations, which describes the technical details on how to compute results in a fast way. 


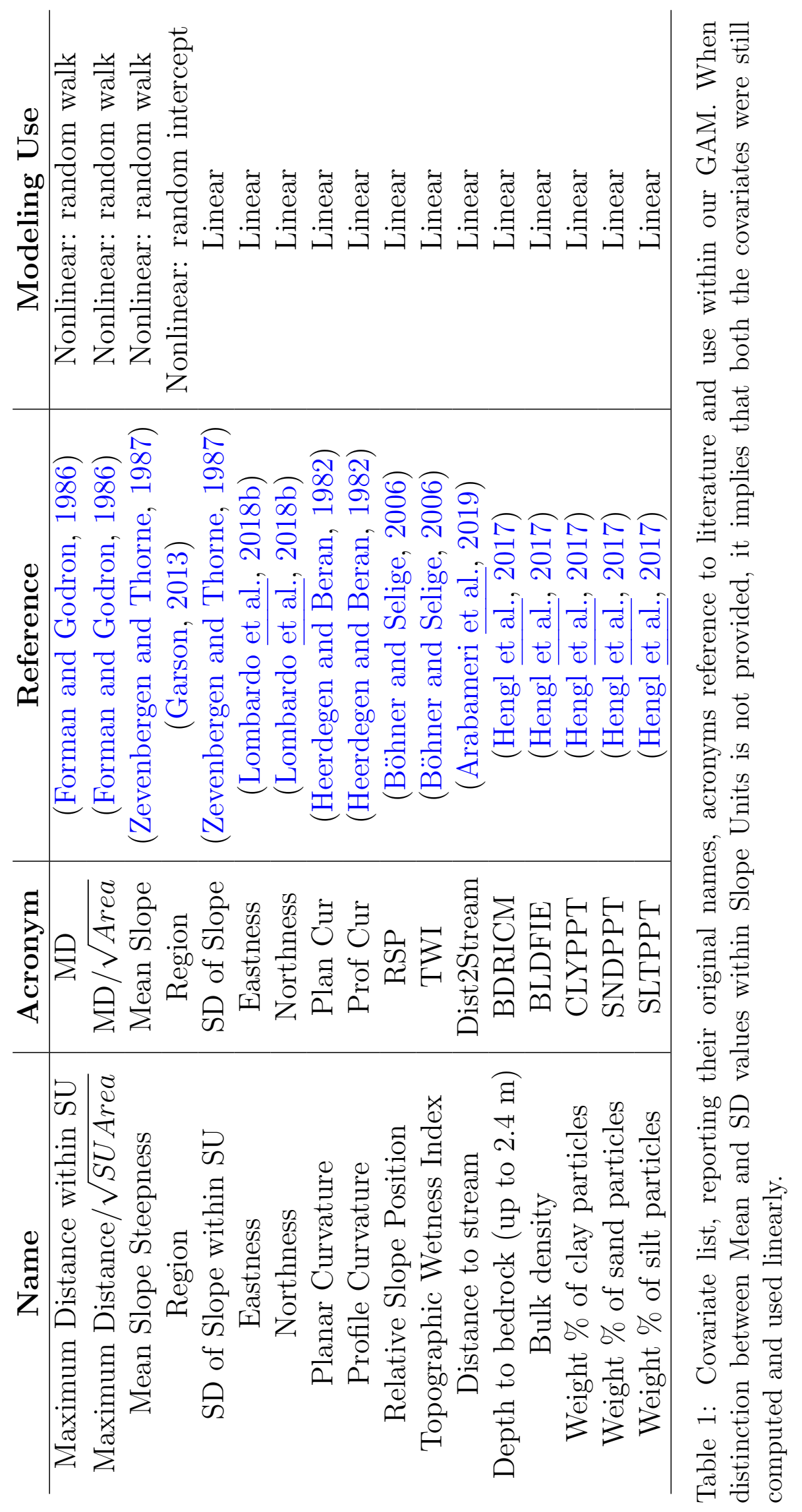




\subsection{Model setup}

We model the presence/absence of landslides $y$ through the Binomial likelihood,

$$
y_{i} \sim \operatorname{Binomial}\left(n=1, p_{i}\right)
$$

where $p_{i}$ is the Binomial probability. We model $p_{i}$ through the frequently used logit link function,

$$
\eta_{i}=\frac{p_{i}}{1-p_{i}},
$$

and refer to $\eta$ as the predictor. The predictor is where we model the relationship between the landslide occurrence and the covariates. We do this by specifying one effect, or model component, per covariate, and then adding these effects together. Let

$$
\eta_{i}=\beta_{1} x_{1}(i)+\ldots+\beta_{m} x_{m}(i)+u_{1}\left(\text { region }_{i}\right)+u_{2}(i)+u_{3}(i)+u_{4}(i),
$$

where $\beta_{j} x_{j}$ are the linear effect, describing the linear relationship of the covariates $x_{j}$ and the predictor. For $\beta_{j}$ we use the default priors in INLA, which are uninformative flat priors.

For $u_{1}$, we specify a random intercept model, called an iid-model in INLA,

$$
u_{1}\left(\text { region }_{i}\right) \sim \mathcal{N}\left(0, \sigma_{u}^{2}\right) .
$$

This means that we estimate one regression constant for each Italian region, independently from each other.

For $u_{2}, u_{3}$, and $u_{4}$ we use the spline known in INLA as the random walk order 1 spline. We have spline models on the covariates $M D$ for $u_{2}, M D / \sqrt{\text { Area }}$ for $u_{3}$, and Mean Slope for $u_{4}$ (see Table 1 for acronyms' reference). For each spline, the covariate is divided into 20 intervals, and the vector of $v_{j}=u_{\text {spline }}\left(\right.$ interval $\left._{j}\right)$ for $j=1, \ldots, 20$, assumes the form

$$
v_{i+1}=v_{i}+\epsilon_{i}
$$

where $\epsilon_{i} \sim \mathcal{N}\left(0, \sigma_{v}^{2}\right)$.

The prior for $\sigma_{u}$ and $\sigma_{v}$ are exponential distributions with mean $\lambda=9.2$, chosen based on the penalising complexity framework by Simpson et al. (2017). In addition the spline has been scaled to give better performance during Bayesian inference, according to Rue and Held (2005).

\subsection{Fit and Cross-Validation procedure}

We first fitted an initial reference model using the whole landslide dataset, separately for each landslide class (type). We did not select a balanced sample, for Petschko et al. (2014); Lombardo and Mai (2018) demonstrated that this operation induces distortions in the global intercept for any susceptibility model. We explored the distribution of the regression coefficients estimated for each region and for each landslide type, and investigated the regions 
for which the intercepts were consistently negative irrespective of the landslide type. We crossed this information with additional sources of information, to evaluate whether there were regions with a manifestly incomplete inventory.

On the basis of the regions we deem to have an incomplete inventory, we run three additional operations, reported below:

- We initially excluded these regions from the analyses, and used the complementary regions, which differ for each landslide type, to calibrate a susceptibility model (biasreduced model). We validated by implementing a 10 -fold cross validation (10-CV), in which each testing subset is mutually exclusive from the remaining nine. In other words, no SU are repeated across CV replicates. This allows one to explore the whole dataset disregarding autocorrelation issues among single CV folds (because same SU may enter different CV-folds).

- Next, we implemented a simulation stage for which we generated a distribution of 1,000 susceptibility estimates for each SU, also for the excluded regions. This simulation phase used the uncertainty estimation obtained from the Bayesian model, ensuring that the uncertainty consistently propagates both in the regions that have rich and poor landslide inventories. Further information on the simulation is in Appendix A.

- Next, we extracted the mean and the $95 \%$ credible interval (CI); the latter is the distance between the $97.5^{\text {th }}$ and the $2.5^{\text {th }}$ percentiles of each distribution. Eventually, we prepared raster maps with the mean susceptibility for each landslide type and its uncertainty, for the whole of Italy.

\subsection{Performance evaluation}

We assessed the performance of the reference model as well as of the bias-reduced models; $c f$. Section 3.3. This was achieved considering threshold-independent and threshold-dependent performance metrics, widely used to assess the prediction skills of binary classifiers.

Specifically, the binomial GAM returns a distribution of estimated probability values for each SU. From each probability spectrum assigned to an SU, we extracted a single value representing the posterior mean. The ensemble of the posterior means extracted from all of the SU also returns a probability distribution, which we used crossing it with the observed landslide presence/absence instances to assess the goodness-of-fit and the prediction skill of susceptibility maps prepared here (Rahmati et al., 2019).

For each landslide type, we took the corresponding probability distribution assigned at SU level and calculated Recevier Operating Characteristics (ROC) curves. These are cutoff-independent metrics because the susceptibility spectrum is binarized many times, each time choosing a different probability threshold. Then, for each value of the cutoff, a pair or values is computed by comparing the observed presence/absence landslide information with respect to the binarized instances. These values consist of False Positive Rate (FPR) 
and True Positive Rate (TPR), from which the ROC curve can be obtained (Hosmer and Lemeshow, 2000). The numerical integral of the ROC curve is the area under the curve (AUC) and represents the deviation of the predictions from random predictions, i.e., a measure of performance.

A similar framework is also valid for the cutoff-dependent metrics, with the difference that the cutoff is single-valued. The confusion matrix obtained by comparing predicted and observed presence/absence instances gives accuracy values for positives and negatives (modeled TP / Observed P, modeled TN / Observed N). We adopted the median posterior mean of the probability as a cutoff for cutoff-dependent metrics. We choose the median instead of the mean (as in Rossi et al., 2010; Lombardo et al., 2016), because our dataset is unbalanced (more slope units flagged with landslide absence than presence), resulting in a posterior mean distribution positively skewed (Frattini et al., 2010) rather than being normally distributed around the mean value, if we had a balanced dataset (same, or comparable, number of landslide absences and presences).

\section{Results}

\subsection{Reference model (within-sample)}

The fitting procedure produced satisfying results with cutoff independent, goodness-of-fit metrics constantly equal or greater than the excellence threshold according to Hosmer and Lemeshow (2000). In Figure 4, we report each ROC curve and AUC value, one for landslide type. The minimum among all types corresponds to $\mathrm{AUC}=0.77$ for Shallow landslides, whereas the maximum is reached for Diffused Fall, with $\mathrm{AUC}=0.92$.

As regards the cutoff-dependent evaluation of the goodness-of-fit, Figure 5 shows that accuracy, for the different landslide types, is spread from a minimum near $85 \%$ of correctly estimated landslide presences found both for Shallow and Translational to a maximum of 97\% for Diffused Fall. These values indicate outstanding goodnees-of-fit performance. As for the capacity of our reference model to label stable SUs, the situation is very different. In fact, the percentage of matching cases between the number of observed and estimated $\mathrm{SU}$ where landslides are absent is relatively low, going from a minimum of around $44 \%$ for Translational to a maximum of $49 \%$ for DSGSD. At a superficial level, this should imply that the model performance are insufficient. However, we need to keep in mind that SU have been delineated by removing near-flat: they all represent rough topographies. As a result, a proportion of correctly predicted absences of approximately $50 \%$ implies that the model assigned a relatively high susceptibility to a large number of cases where the current observation is for these processes not to be there. However, this does not mean that they won't occur in the future (or have already occurred but have not been identified and included in the inventory), hence the high susceptibility estimates, which is a very reasonable situation in a territory that has been suffering from widespread landsliding as long as these surface processes have been recorded (Rossi et al., 2019). 

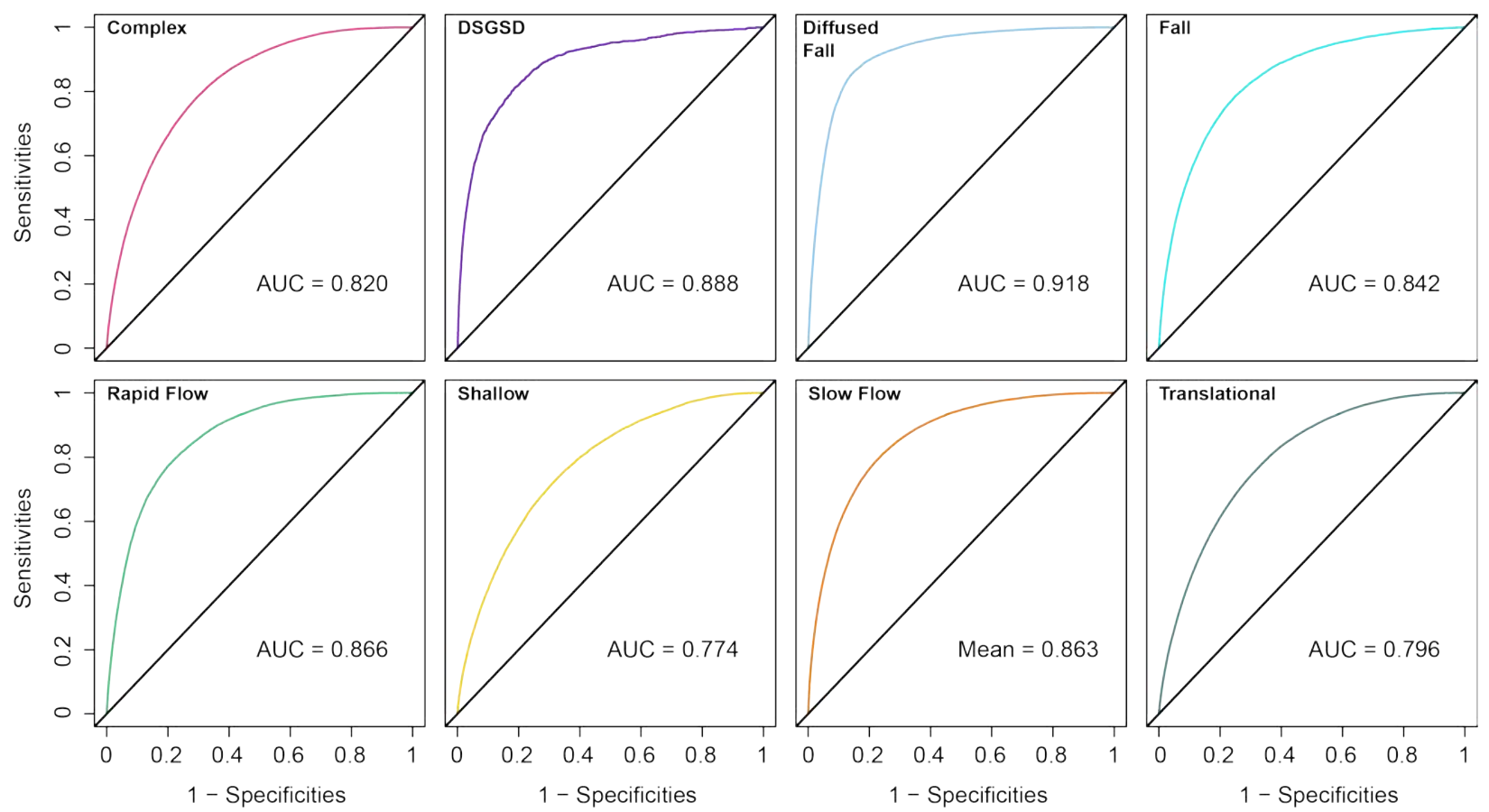

Figure 4: Goodness-of-fit summary of the reference models built for each landslide type. 


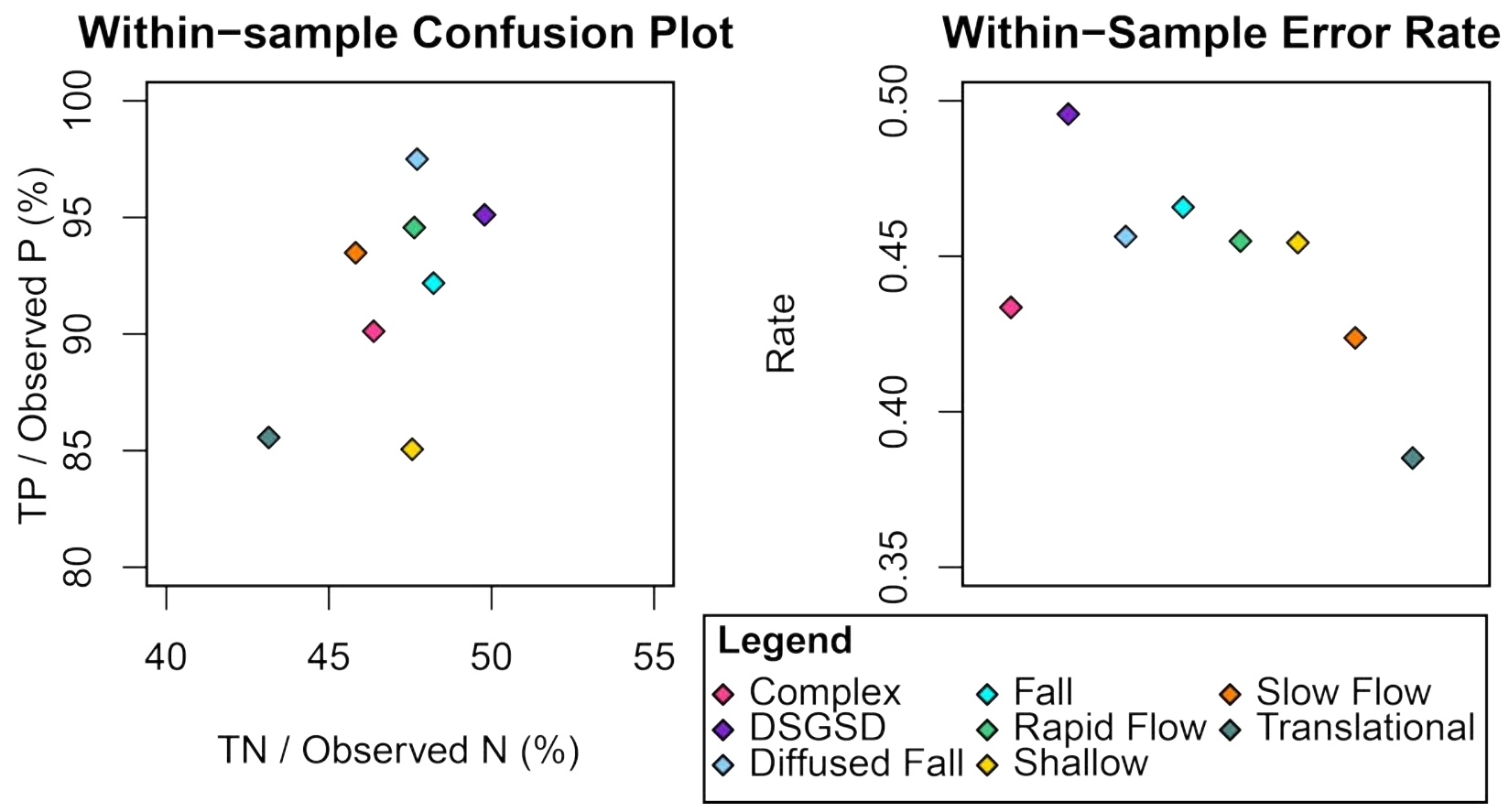

Figure 5: The left panel shows the confusion plot (see Lombardo et al., 2015), constructed via the percentage of Observed TP and fitted TP against the percentage of Observed TN and fitted TN (for each landslide type). The right panel reports the error rates (for each landslide type). 


\subsubsection{Fixed Effects}

Some interesting patterns arise examining the linear components (cf. Section 3.2) included in our approach. Figure 6 shows the posterior marginal distributions of each covariate assumed as a linear effect and for each landslide type. Specifically, we displayed the covariates for which the marginal distribution was significant 2.5 and 97.5 percentiles of the regression coefficient distribution share the same sign for at least one landslide type. The figure summarizes one of the main strengths of a Bayesian susceptibility implementation, for regression coefficients are assigned their posterior mean and its associated uncertainty measured as the $95 \%$ credible interval.

The fixed effects change in sign and amplitude for different landslide types. And, for landslide type that share some degree similarity, this is much less pronounced than for landslide types with a completely different failure mechanism.

For instance, the fixed effects estimated for Fall and Diffused Fall often appear to overlap while markedly differing from Flows and Shallow mass movements. This is the case for Mean Northness where both the posterior distribution of Fall and Diffused Fall are located to the left side of the plot and share a negative regression coefficient, respectively centered at approximately -0.06 and -0.12 . Conversely, Translational and Slow Flow were estimated with a positive regression coefficient, respectively centered at around 0.08 and 0.1 . These results look reasonable as falls may be influenced by large temperature variations related to the southern orientation (Loche et al., 2021), while Translational movements and Slow Flow may be positively correlated with higher soil moisture, which is favoured by lower solar radiation. Another striking example can be seen in $S D$ of Slope for which the regression coefficient of Fall and Diffused Fall is positive; the existence of a cliff, where these landslides typically occur, implies a large variation in slope steepness within an SU. On the contrary, all the other landslide types are either not affected or even negatively affected by the variation of slope steepness. This is the case for DSGSD, a landslide type with a posterior mean centered at zero, for which the buried failure surface may not be sensitive to variations at the surface. And it is also the case of Rapid Flow, Shallow, Slow Flow and Translational, which share a negative regression coefficient, likely due to the fact that rough SUs may host internal barriers opposing the initial failure initiation movement. Such consideration has been reported already in the literature. For instance, Tanyas et al. (2017) showed that frequency of landslides are higher for low roughness values, hence for low SD of Slope. They observed that the frequency proportionally decreases for increasingly rougher topographies, and they justified this observation by assuming that roughness may be a proxy for rocky outcrops, where low $S D$ of Slope implies softer surface materials or soils and high $S D$ of Slope implies rocks or just material with higher geotechnical strength.

A similar situation, where predominantly superficial landslide behave consistently, exists for the regression coefficients estimated for the mean bulk density (BLDFIE). In this case, Translational, Slow Flow, Shallow and Complex landslides all share a positive marginal effect of BLDFIE on landslide susceptibility (Adams and Sidle, 1987). 

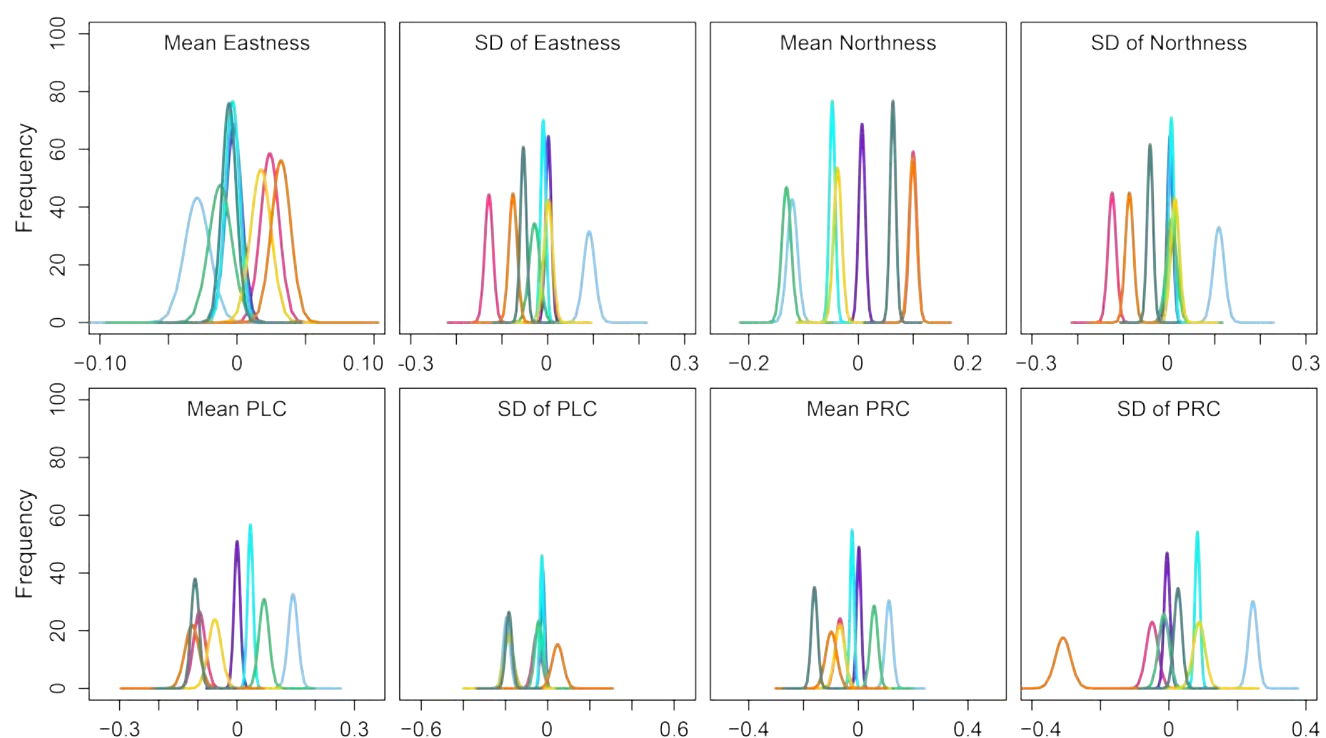

Legend

$\Lambda$ Complex

$\wedge$ DSGSD

$\Lambda$ Diffused Fall

1 Fall

A Rapid Flow

A Shallow

^ Slow Flow

$\Lambda$ Translational
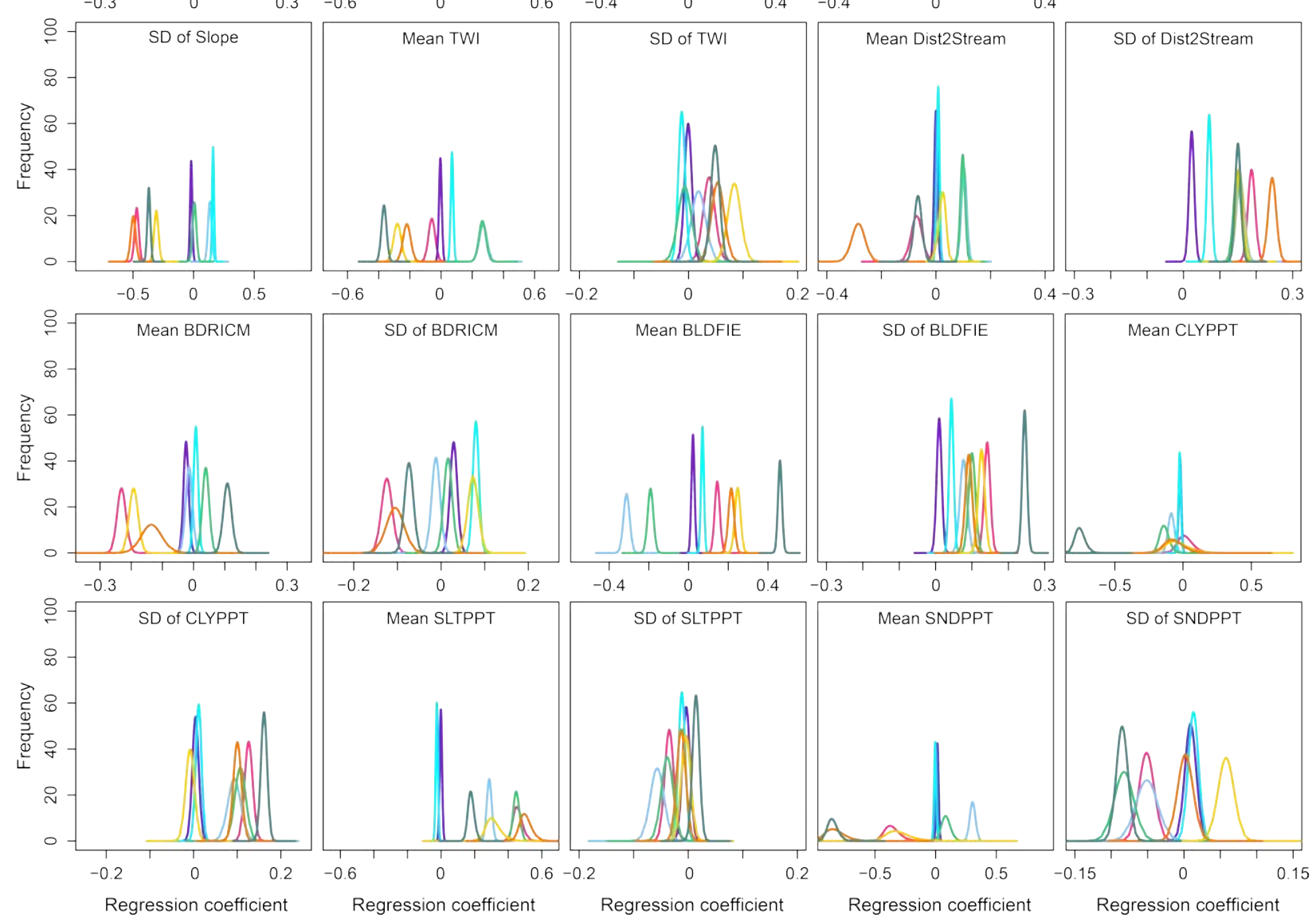

Figure 6: Fixed effects expressed as marginal distributions for each landslide type. 
Clearly, this level of straightforward interpretation does not apply to every fixed effect and every landslide type. In such a complex model, most of the estimated fixed effect are geomorphologically reasonable and, most importantly, lead to excellent goodness-of-fit performance.

\subsubsection{Random Effects with adjacent-class-dependency}

In this section we present a summary of the random walk effects. We remind, here, that we applied a random walk to ensure that $M D, M D / \sqrt{\text { Area }}$ and Mean Slope would retain the ordinal structure of their original continuous distribution ( $c f$. Section 3.2 for definitions).

In Figure 7, $M D$ (or the maximum distance within an SU) appears to behave nonlinearly, justifying the choice of the their use as random effects. Looking at the eight trends, it becomes clear that high susceptibility values correspond to large values of the slope units length. However, it is also evident that Complex, Rapid Flow, Slow Flow and Translational have a marked (near exponential) increase in their respective regression coefficient for $M D$ values greater than 10,000 m. Conversely, DSGSD, Diffused Fall, Fall show a much milder trend, with Shallow being the only landslide type in between the other two groups.

We can give a geomorphological interpretation for the observations described above. In fact, complex/translational movements, slow and rapid flows can be large in size and need relatively large slopes (long, or wide) to occur. Falls and diffused falls can also occur on small slopes. DSGSD mainly depends on the presence of tectonic discontinuities, unloading of glacier retreat and seismic activity, thus being relatively less related to slope size and local morphology and more related to conditions that involved fully-coupled thermo-hydromechanical behaviour of the materials (Segui et al., 2020; Scaringi and Loche, 2022).

In Figure $8, M D / \sqrt{\text { Area }}$ (or the elongation/roundness index of each SU) also appears to behave nonlinearly. Similarly to the previous random effect, the behavior of the SU elongation appears to have some degree of consistency across certain landslide types. DSGSD, Diffused Fall, Fall, Rapid Flow and to some extent also Shallow. In these cases, the effect of $M D / \sqrt{\text { Area }}$ is negligible up to a threshold $M D / \sqrt{\text { Area }}=4$ (we recall here that this index is dimensionless) after which at increasingly elongated SUs the probability of the corresponding landslide type would drastically increase.

Elongation of the slope units can be in the direction of the surface drainage, or even perpendicular to that. We observe that Rapid Flow and DSGSD can be correlated with SUs parallel to the drainage, while wide and short, steep slopes can accommodate mainly Diffused Fall and Fall.

Conversely, Complex, Slow Flow and Translational landslides share a common behavior and appear to correlate poorly with elongation of the slope units. We conclude that these types of landslides mainly occur inside large semi-circular slopes.

The last covariate modeled with a random walk is Mean Slope, for which we also found a nonlinear influence on the estimated susceptibility, irrespective of landslide type. As in the previous cases, more than one landslide type behaves similarly to others. DSGSD and 


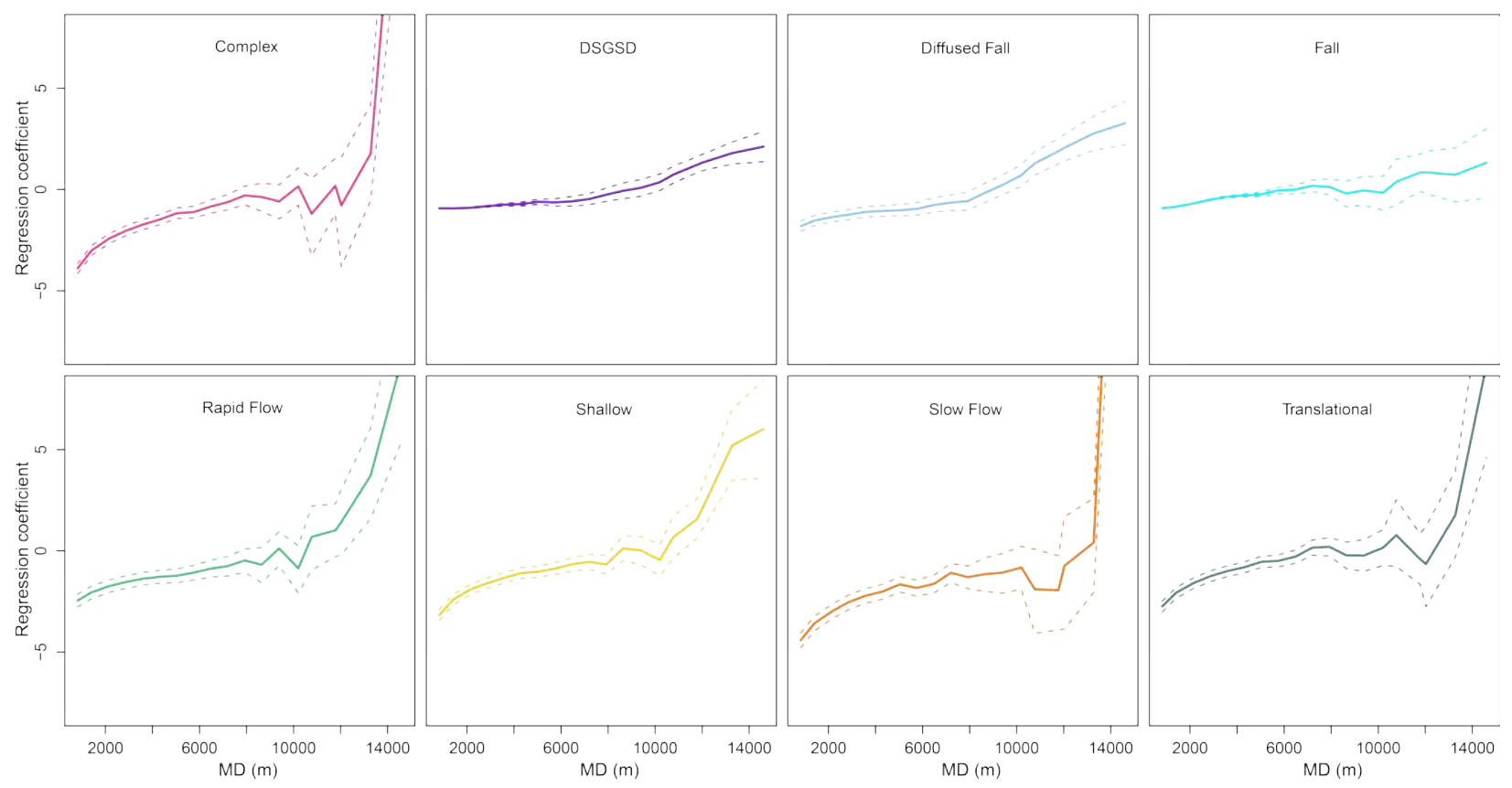

Figure 7: Maximum distance within an SU effect on each landslide type susceptibility. The effect is modeled as a random effect estimated over 20 classes with adjacent dependency. Thick colored lines represent the posterior means whereas the colored dashed lines indicate the posterior 95\% credible interval. Dashed grey lines indicate the zero line along which coefficients play no role with respect to the modeling outcome. 


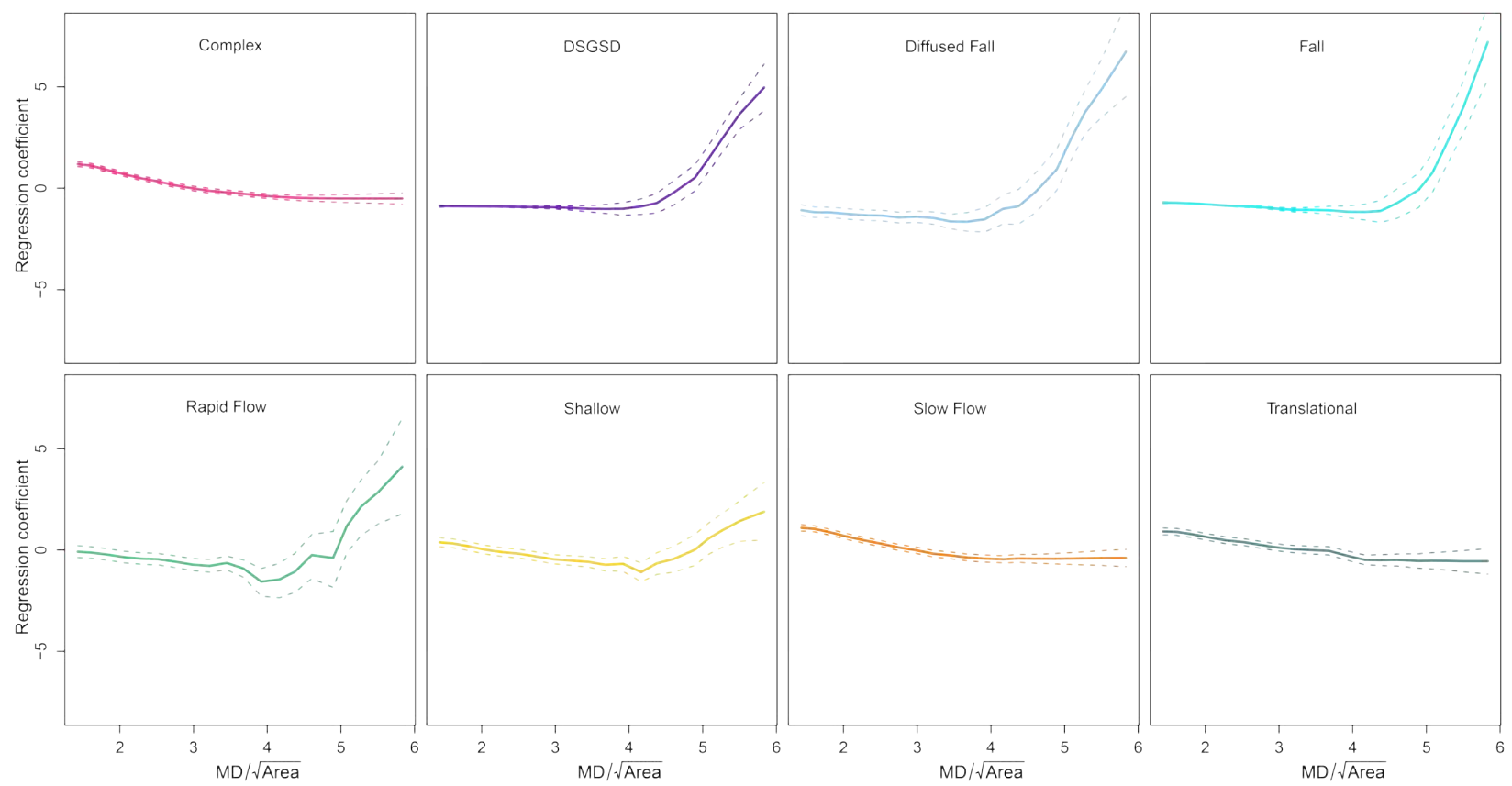

Figure 8: Maximum Distance/ $\sqrt{A r e a}$ (roundness/elongation) effect on each landslide type susceptibility. The effect is modeled as a random effect estimated over 20 classes with adjacent dependency. Thick colored lines represent the posterior means whereas the colored dashed lines indicate the posterior $95 \%$ credible interval. Dashed grey lines indicate the zero line along which coefficients play no role with respect to the modeling outcome. 


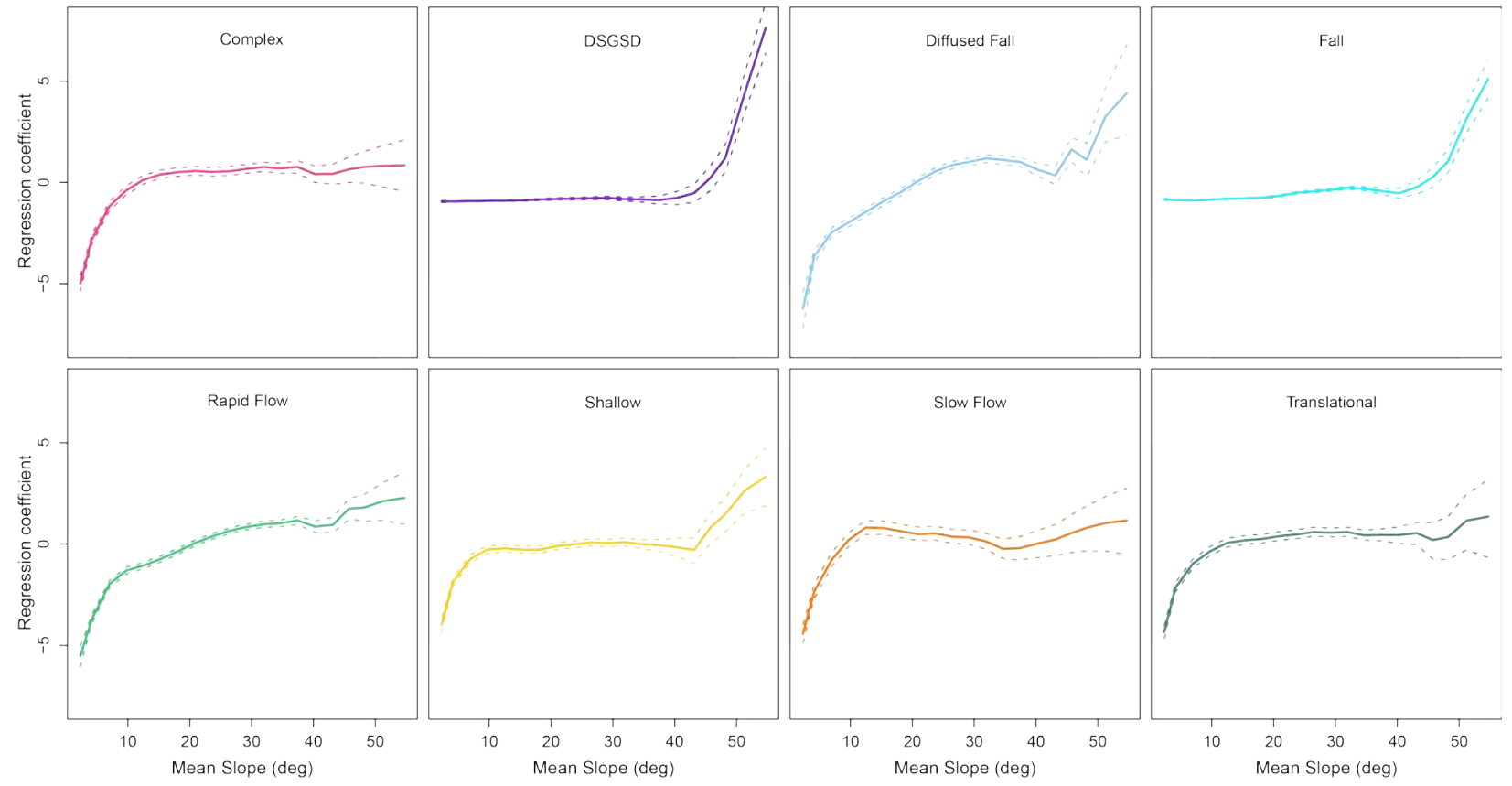

Figure 9: Mean Slope effect on each landslide type susceptibility. The effect is modeled as a random effect estimated over 20 classes with adjacent dependency. Thick colored lines represent the posterior means whereas the colored dashed lines indicate the posterior $95 \%$ credible interval. Dashed grey lines indicate the zero line along which coefficients play no role with respect to the modeling outcome. 


\subsubsection{Random Effects with multiple regional intercept}

In this section we present results obtained using a multiple intercept approach, i.e. calculating an intercept for each region, which helped to asses the level of completeness of the regional landslide inventories.

Figure 10 shows each multiple intercept. The characteristic that stands out the most is that the credible intervals are extremely narrow, irrespective of landslide type. We observe that the value of the multiple intercept changes significantly, for the same region, when different types of landslides are considered. We also note that for some regions, as Piedmont (PIE), Lombardy (LOM) and Liguria (LIG), coefficients are almost always positive, while for Sardinia (SAR) and Apulia (PUG) they are frequently negative. Grey dashed lines in the plots correspond to the zero reference level below which a negative correlation between landslides presence and administrative region exists. Reasons for this negative correlation may be geomorphological (a given type of landslides is not expected in a given region), or caused by the scarce quality and completeness of the regional inventory. Section 4.2 illustrates additional criteria to decide which region had incomplete landslide inventories.

\subsection{Inventory completeness/incompleteness considerations}

To understand which regional inventory could be considered complete at a sufficient level, we revised the inventories through random heuristic checks, examined the information provided in technical reports (see here for regional reports and here for the national report), and combined this qualitative expert knowledge together with more quantitative considerations driven by data displayed in Figures 10 and 11 . 


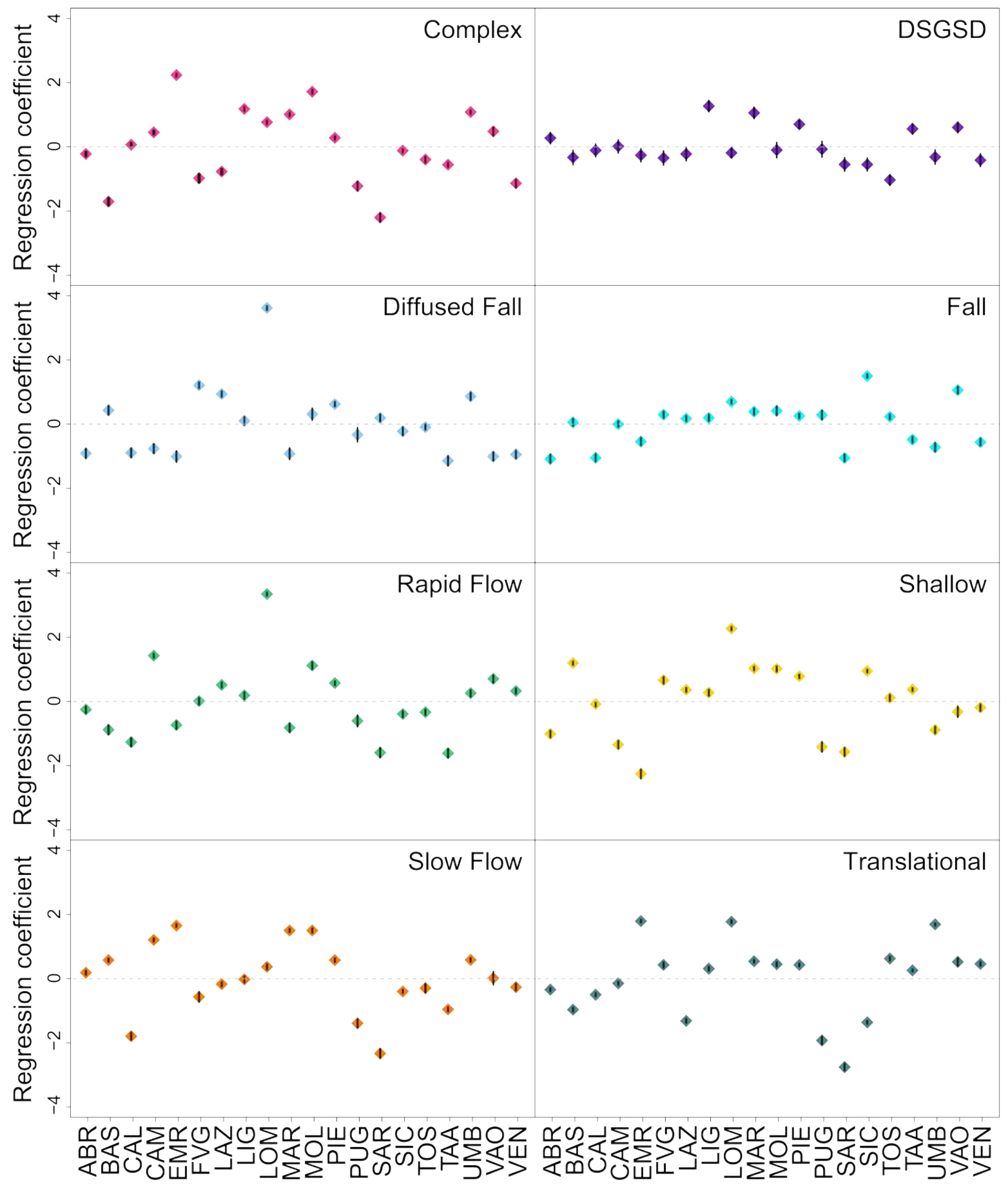

Figure 10: Posterior distribution of the multiple regional intercepts for each landslide type. Because the estimated uncertainty is particularly small, the posterior mean values are shown as diamonds whereas the $95 \%$ credible intervals are depicted as black vertical bars. 


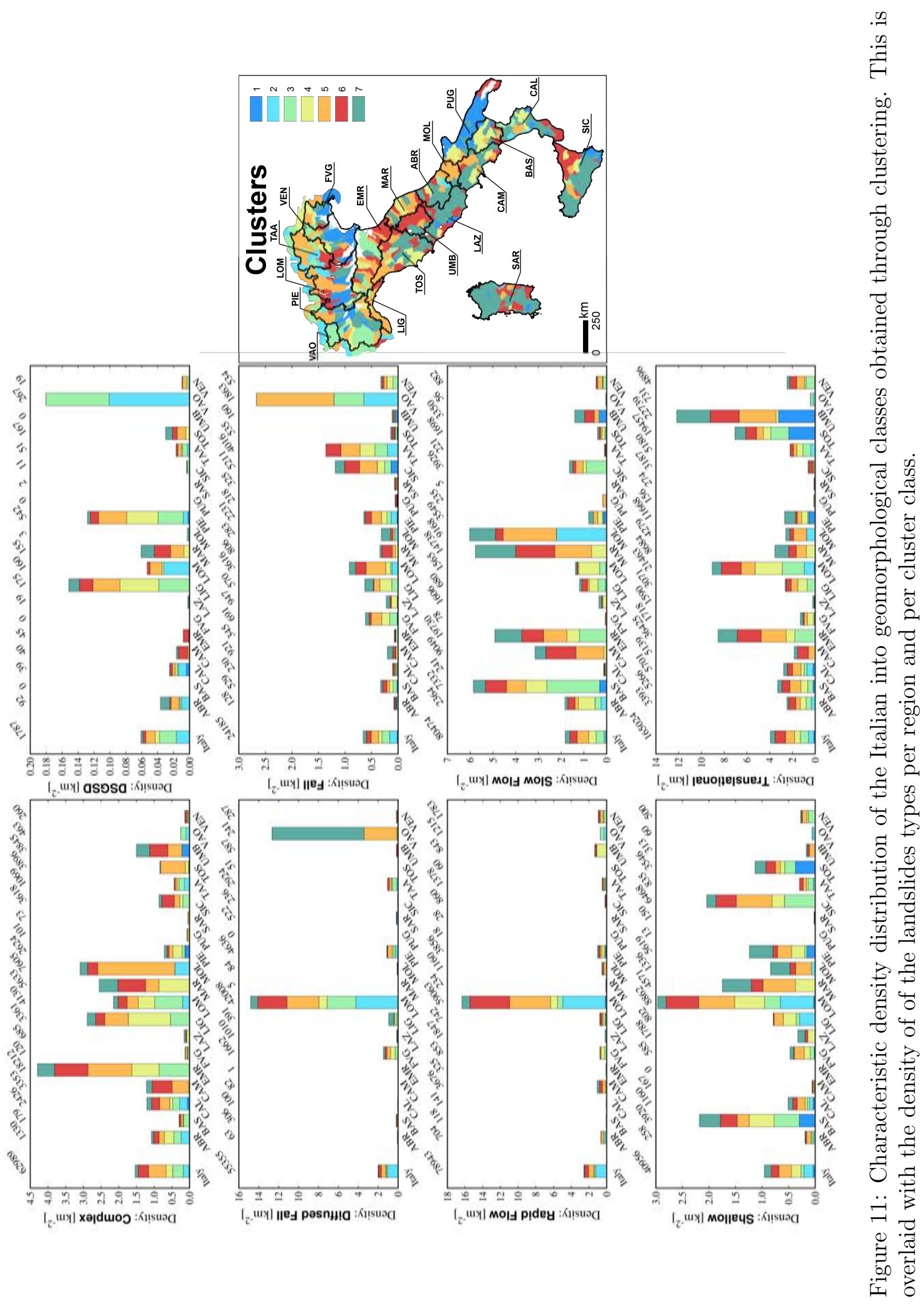


Figure 11 includes the map resulting from the spatial geomorphological clustering proposed by Alvioli et al. (2020). The seven clusters are representative of geomorphologically and lithologically homogenous conditions across Italy and they are based on the very same SU partition used in this work. From a landslide perspective (including the eight IFFI types), we should expect an analogous signal of landslide densities per clusters, irrespective of the region at hand. This is confirmed, for example, by comparing, at cluster level, the densities of Slow Flow in Basilicata (BAS, southern Italy) with those in Emilia Romagna (EMR, Northern Italy) or the densities of Fall in Sicily (SIC, southern Italy) with those in Trentino Alto-Adige (TAA, Northern Italy). The comparison confirms that in areas that share the same characteristics from a morphological and geological point of view, the density of landslide phenomena of the same type is at least comparable. Thus, overall we considered an indication for a potentially incomplete inventory any strong deviation from the landslide density distribution in the clusters' polygons, associated with a strong negative intercept in Figure 10 and through heuristic checks and report descriptions. The results are summarized in Table 2, where the teal cells and red cells indicate, respectively, reliable inventories and incomplete inventories and numbers represent the mean value of the multiple intercept values.

Table 2: Values of the multiple intercept for the different regions and landslide types. The teal colorcode corresponds to regions that appeared consistent in terms of landslide densities per geomorphological clusters (see Alvioli et al., 2020) and multiple intercept. The red color indicates a significant deviation from this trend and thus we consider it an indication for a incomplete regional inventory. In other words, for the next modeling procedure, we used the teal region for training and the red regions for model transferability.

\begin{tabular}{|c|c|c|c|c|c|c|c|c|}
\hline Regions & Complex & DSGSD & Diffused Fall & Fall & Rapid Flow & Shallow & Slow Flow & Translational \\
\hline ABR & -0.22 & 0.28 & -0.75 & -0.70 & -0.25 & -1.01 & 0.19 & -0.34 \\
\hline BAS & -1.71 & -0.33 & 0.08 & -0.05 & -0.88 & 1.20 & 0.58 & -0.96 \\
\hline CAL & 0.07 & -0.11 & -0.78 & -0.72 & -1.27 & -0.08 & -1.79 & -0.50 \\
\hline CAM & 0.45 & 0.02 & -0.67 & 0.41 & 1.43 & -1.34 & 1.21 & -0.14 \\
\hline EMR & 2.24 & -0.26 & -0.91 & -0.52 & -0.73 & -2.25 & 1.66 & 1.80 \\
\hline FVG & -0.98 & -0.35 & 1.35 & 0.66 & 0.01 & 0.67 & -0.57 & 0.43 \\
\hline LAZ & -0.77 & -0.22 & 1.06 & 0.55 & 0.52 & 0.37 & -0.17 & -1.31 \\
\hline LIG & 1.18 & 1.27 & 0.17 & 0.26 & 0.19 & 0.28 & -0.02 & 0.31 \\
\hline LOM & 0.77 & -0.19 & 3.88 & 1.09 & 3.34 & 2.28 & 0.37 & 1.78 \\
\hline MAR & 1.01 & 1.06 & -0.83 & 0.87 & -0.82 & 1.03 & 1.50 & 0.54 \\
\hline MOL & 1.72 & -0.10 & 0.39 & 0.75 & 1.12 & 1.02 & 1.50 & 0.45 \\
\hline PIE & 0.28 & 0.71 & 0.87 & 0.60 & 0.58 & 0.79 & 0.58 & 0.43 \\
\hline PUG & -1.22 & -0.07 & -0.26 & 0.56 & -0.60 & -1.41 & -1.39 & -1.93 \\
\hline SAR & -2.20 & -0.55 & 0.31 & -0.75 & -1.60 & -1.57 & -2.33 & -2.76 \\
\hline SIC & -0.12 & -0.55 & 0.51 & -0.31 & -0.39 & 0.95 & -0.40 & -1.36 \\
\hline TAA & -0.39 & -1.03 & -1.92 & -0.79 & -0.33 & 0.12 & -0.29 & 0.63 \\
\hline TOS & -0.55 & 0.56 & -1.02 & -0.12 & -1.61 & 0.38 & -0.96 & 0.26 \\
\hline UMB & 1.09 & -0.32 & 1.02 & -0.37 & 0.26 & -0.88 & 0.59 & 1.69 \\
\hline VAO & 0.48 & 0.61 & -1.70 & -1.29 & 0.71 & -0.32 & 0.02 & 0.52 \\
\hline VEN & -1.13 & -0.41 & -0.78 & -0.12 & 0.33 & -0.19 & -0.26 & 0.46 \\
\hline
\end{tabular}

A quick example of the selection procedure can be taken from the analysis of the plot (11) 
concerning Shallow landslides. The total height of the bars depends on the landslide density measured in individual clusters, represented with the same colors as in Alvioli et al. (2020). Data show that Shallow landslides occur quite homogeneously in all of the different clusters (apart from a scarce presence in cluster 1). This is confirmed by data of many regions (including BAS, LOM, SIC, CAL, TOS) where, despite the total densities can be different, the ratio between the densities in the different clusters remains quite constant and comparable to the national average. We interpret this behaviour as an indication that surface landslides were at least mapped in these regions. However in other regions (EMR, PUG, SAR, VAO and CAM), information about shallow landslides is very scarce or absent (on all clusters). Since in these regions the values of the multiple coefficient are also negative or very negative, we considered them affected by significant problems of completeness and quality of the shallow landslides inventory. To support this statement, Figure (11) also reports the number of landslides in the top horizontal axis (note that the count of landslides for EMR is zero).

\subsection{Final fits and simulations}

After selecting the regions for which the inventory appeared incomplete, for each landslide type, we fitted a binomial GAM framework on the complementary regions. To test it, we run two complementary procedures. On the one hand, we fitted once again the same models as before (i.e., same covariates, same choice of linear and non-linear effects) but constraining them solely on the regions that we deemed to have a complete, or at least representative, landslide inventory, for each landslide type. This operation ensures the ability to simulate over the regions with incomplete inventories (for more details, see Appendix A). On the other hand, we also performed a standard 10-fold cross-validation procedure using the regions with complete inventories. This operation ensures that we can assess our out-of-sample predictive skill, still within regions where the quality of landslide data is considered reliable.

Below, we present the performance, first, and the simulations, later, illustrated with maps.

\subsubsection{Cross-validation performance}

In analogy to the information provided for the reference model, we summarized the ROC curves and their AUC for each landslide type, through a 10-fold CV. Figure 12 reports 10 ROC curves, and the corresponding AUC variability. The out-of-sample performance occupies a range between acceptable $(0.7<\mathrm{AUC}<0.8)$ and excellent $(0.8<\mathrm{AUC}<0.9)$ binary discrimination, according to Hosmer and Lemeshow (2000), with a minimum mean AUC estimated for Translational landslides at $\mathrm{AUC}=0.766$ (and a very low deviation measured in 0.004 standard deviations). This value is significantly distant from the lower end of the acceptable range and it is actually close to the outstanding one. Similarly, the maximum mean AUC corresponds to $\mathrm{AUC}=0.887$ (0.013 standard deviations $=0.013)$. It 

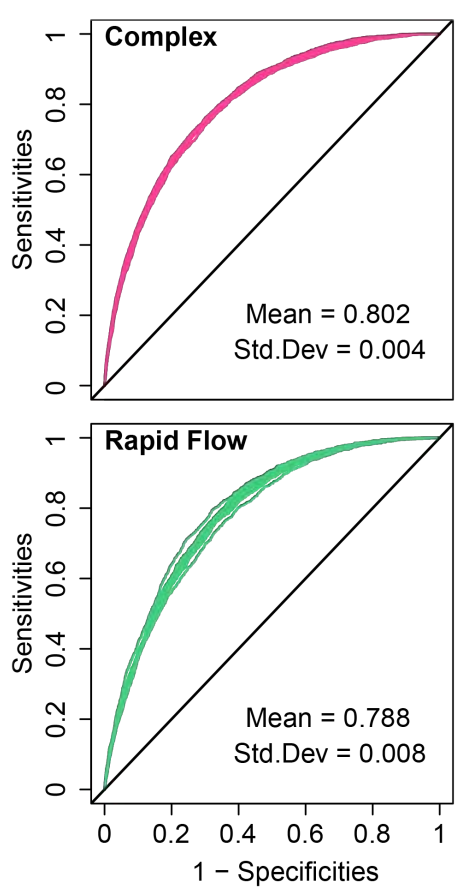

was estimated for DSGSD and it is close to the outstanding performance class limit $(0.9<$ AUC < 1.0). This overview highlights suitable and robust out-of-sample performances for models trained within regions where landslide information is at its best within Italy.
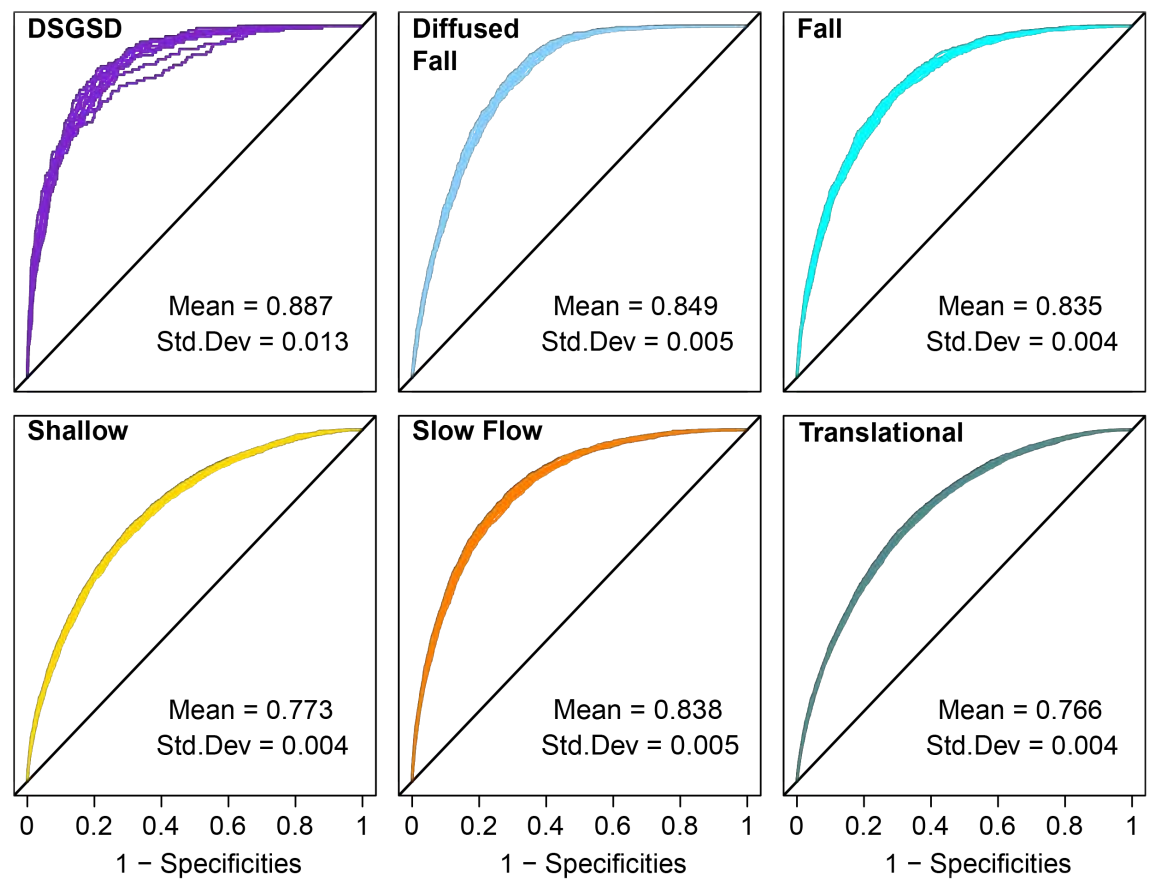

Figure 12: Prediction skill summary obtained from a 10-fold CV run for a set regions which we assumed have a complete landslide inventory, for each landslide type.

Nevertheless, ROC curves and AUC values only provide a lumped overview of model performances, where the returned value is independent from the probability cutoff one may choose. Thus, in analogy to the information provided for the reference model, we also computed the confusion matrix for each of the ten CVs, setting the probability threshold at the posterior median probability. The results, shown in Figure 13, exhibit an interesting behavior, in the reference case. Binomial GAM is able to single out very efficiently SU where landslides occurred. This is proved by very high percentages of TP / Observed P, always above $80 \%$, irrespective of landslide type. However, crossing the estimated probabilities with the observed absences, the model seems to perform poorly, both in terms of TN / Observed N and in terms of Error Rates. This is a crucial point for us to be shared, for we need to recall that the Slope Unit partition used here does not include any flat or near-flat conditions. Therefore, it is specific of rough landscapes where landslides may well occur in the future, but they have just not been observed yet. This is the reason for the discrepancy between estimated probabilities at locations (SUs) and the observed notion of stable mapping units collected so far. In other words, when the percentage of TP / Observed P is confined between $38 \%$ and $50 \%$, irrespective of the landslide type, this implies that our susceptibility models have deemed the complementary $62 \%$ and $50 \%$ of the examined territory to be prone to slope failures. 


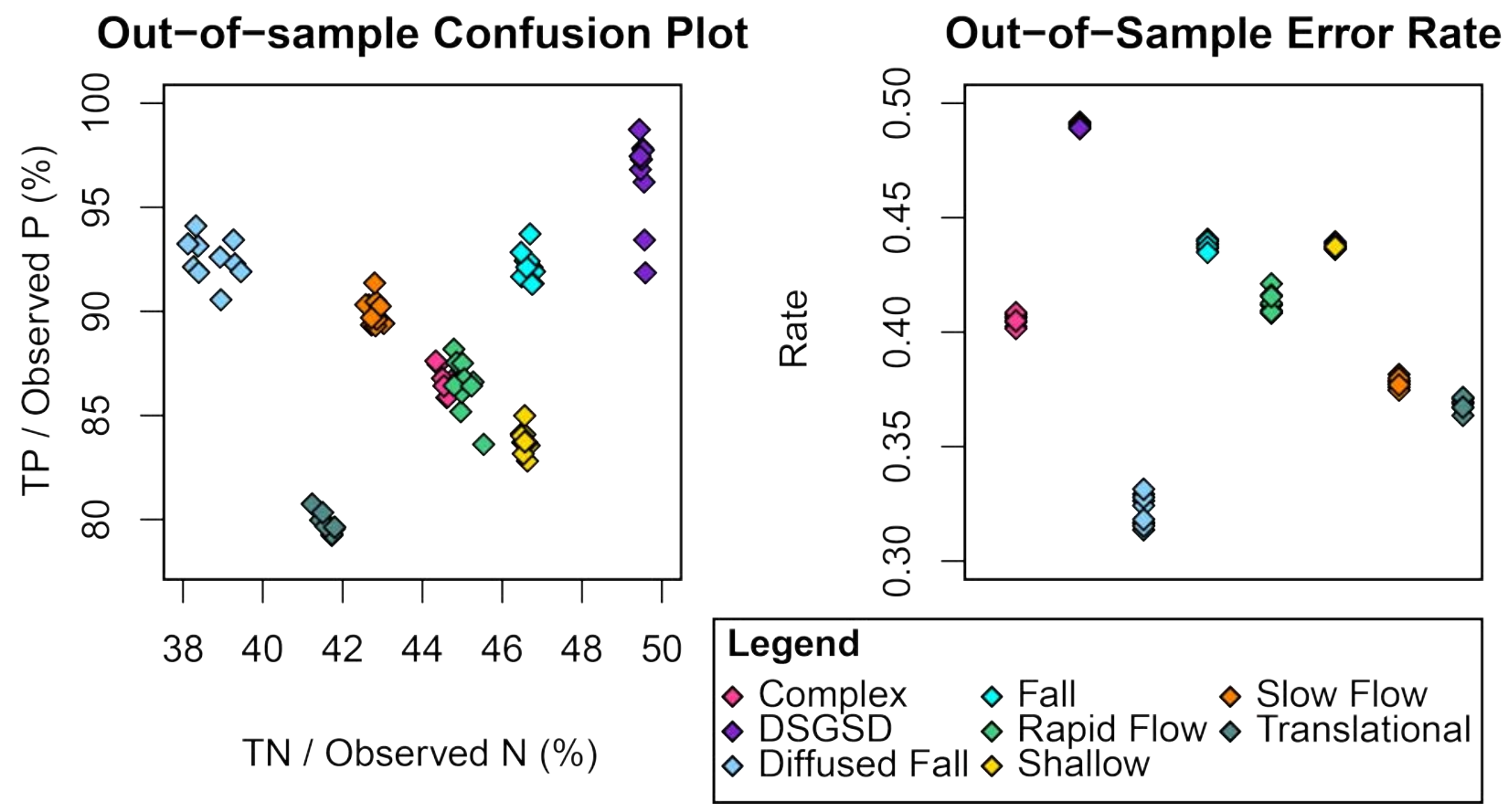

Figure 13: The left panel shows the confusion plot (see Lombardo et al., 2015), constructed via the percentage of Observed TP and fitted TP against the percentage of Observed TN and fitted TN (for each landslide type). The right panel reports the error rates (for each landslide type). This plot has been obtained from a 10-fold CV run for a set regions which we assumed have a complete landslide inventory, for each landslide type. 


\subsubsection{Simulations for susceptibility mapping}

Figures 14 and 15 show maps with the results of simulations (cf. Section 3.2). The former corresponds to the mean of the 1,000 simulations generated for each landslide type and for each SU. The latter is the width of the $95 \%$ CI uncertainty around the mean susceptibility estimates. These two elements represent the variability in how likely a certain landslide type may occur across the Italian territory. Examining Figure 14 one can clearly see the relative dominant pattern of Diffused Fall, DSGSD, Fall and Rapid Flow types over the Alps. This is a particularly interesting result because we did not use a strict spatial model. In fact, a spatial model would treat close SUs more similarly than it would do for SUs that are far apart, because it would be informed of the spatial location of those mapping units. On the contrary, the only element that drives spatial dependence in our model is the value assumed by the covariates we chose. Nevertheless, even if the model is not technically a pure spatial model, the way it characterizes the Alps consistently highlights the highest susceptibility estimates for the three landslide types mentioned above. This is a geomorphologically sound result, which well aligns with another observation. In fact, for the Complex, Shallow, Slow Flow and Translational types, the dominant susceptibility pattern in each map corresponds to the Appenine belt.

\section{Discussion}

Most of the studies of landslide susceptibility existing in the literature typically takes landslide inventories and rely uncritically on them to fit data-driven models. These are often built without questioning their completeness/incompleteness nor the implications that one or the other would lead to in terms of probabilistic results. This is not the case for a relatively small number of contributions (Steger et al., 2016b; Lima et al., 2021; Lin et al., 2021; Steger et al., 2021; Pokharel et al., 2021) where the bias induced into the susceptibility estimates by incomplete inventories is rigorously researched in depth. However, even the authors mentioned above, have not examined regional biases to the extent we propose here. Our work takes deep inspiration from the papers cited above, and extends on the framework they propose by first introducing a spatially-varying regression constant examined per regional administration.

On the basis of the full distribution of the estimated regression coefficients per region and per landslide type, we carried out an extensive search, both qualitative and quantitative, to select best locations to train a susceptibility model (GAM) and transfer the resulting predictive function onto areas characterized by poor landslide inventories. The choice of a Bayesian framework also provides further insight into the full posterior distribution per landslide type, allowing for simulating landslide occurrences with a rich probabilistic description, summarized through the mean behavior and its uncertainty. In turn, this allows to provide end users of the susceptibility assessment with a full suite of information upon which they can make decisions. In fact, knowing if a given slope is likely to be unstable on average 


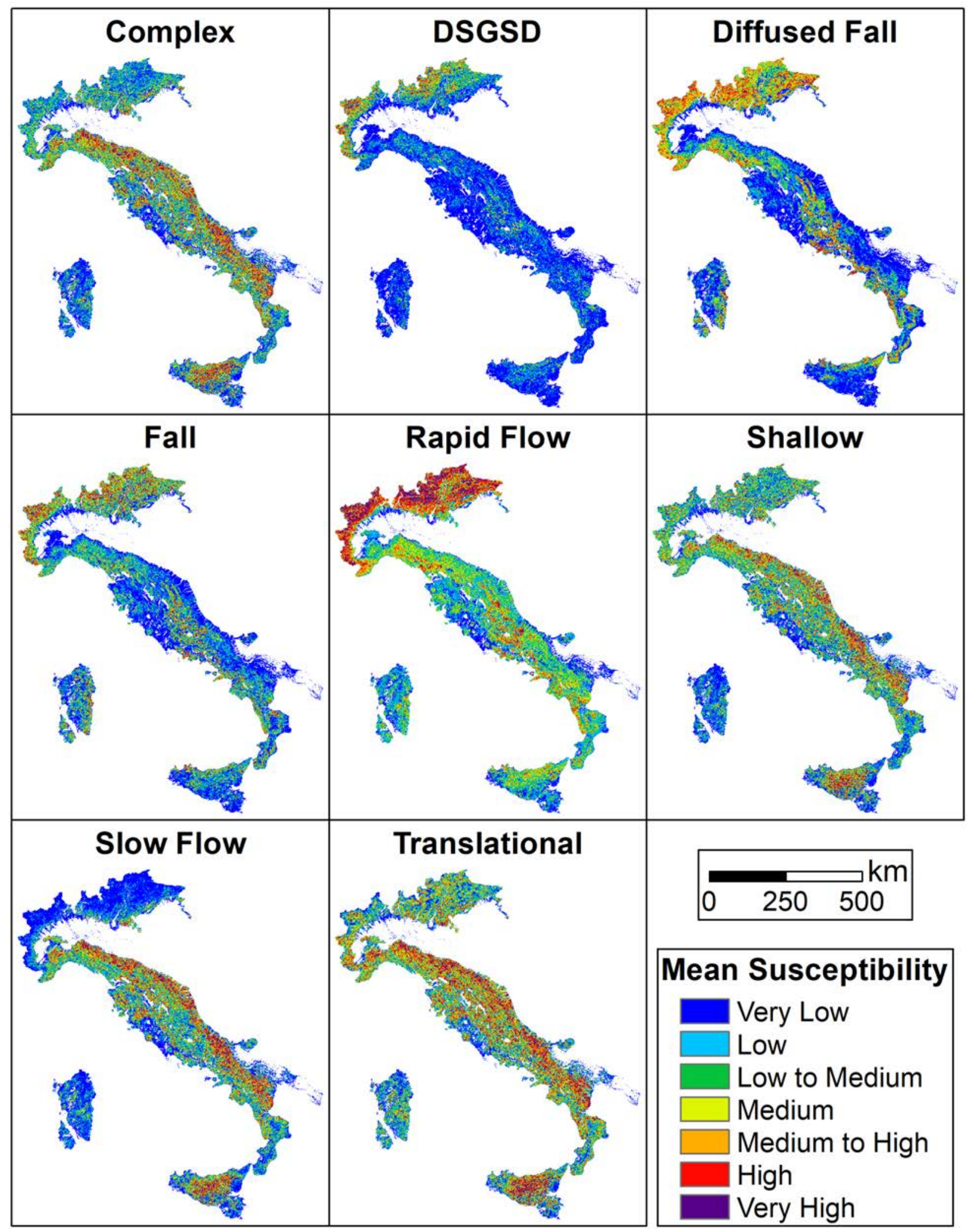

Figure 14: Mean simulated susceptibility maps per landslide type. 


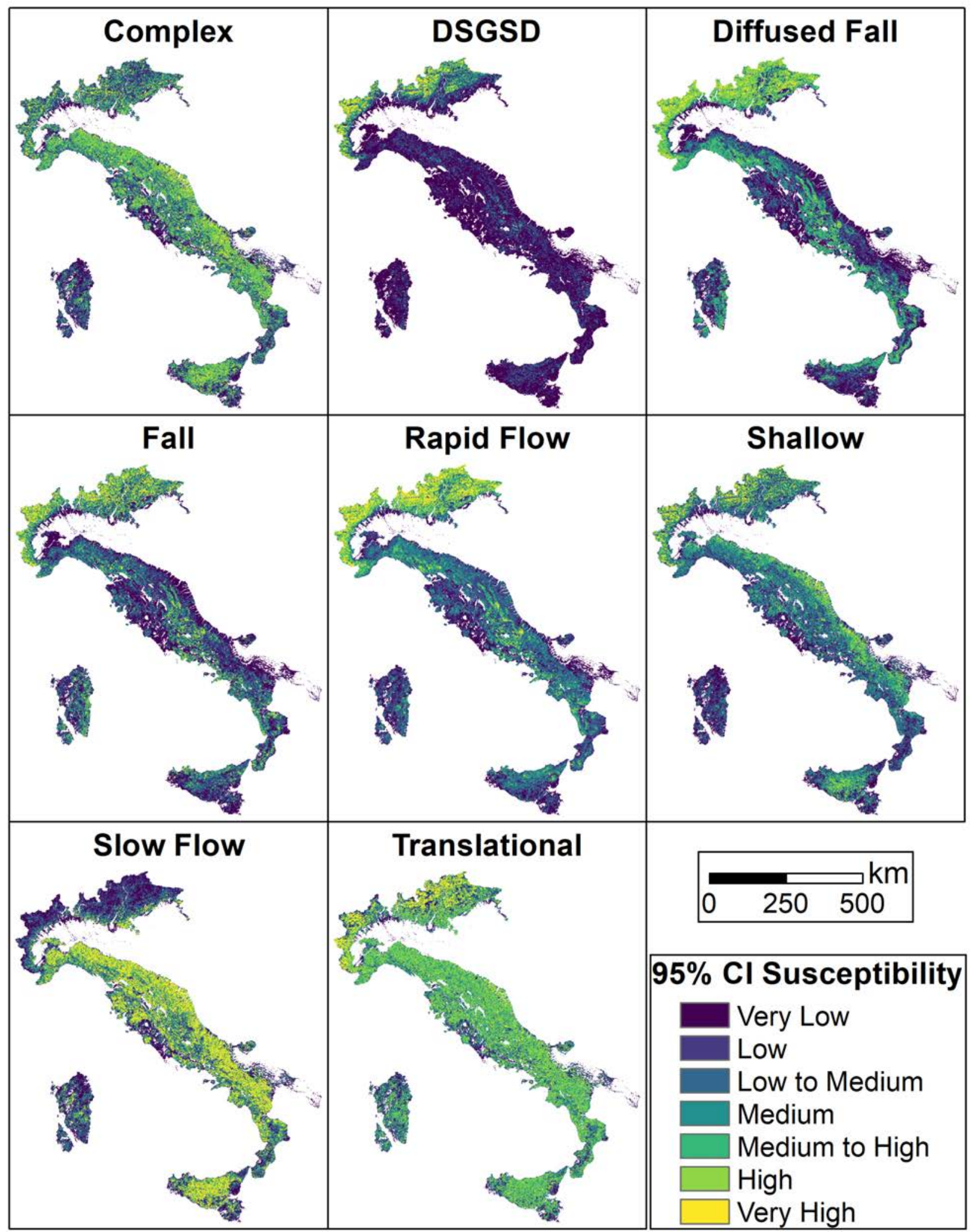

Figure 15: Uncertainty measured with a $95 \%$ credible interval of the simulated susceptibility maps, one per landslide type. 
does not tell the whole story. It is the combination of this information together with the uncertainty level that ensures a much more reliable decision. A slope with a high mean probability of landslide occurrence but with an extremely large uncertainty may not be the right investment for slope stabilization practices. On the contrary, a slope with high mean probability of landslide occurrence, but lower than the ideal one mentioned above, associated with very small uncertainty, may be a safer target for stabilization investments. The same is valid in the opposite situation, a slope with a very low mean susceptibility but with very high uncertainty should not be overlooked, whereas one could safely consider situations where the posterior mean and uncertainty in the susceptibility estimates are both small.

We recall here that the GAM model we fitted, at the Slope Unit (i.e., hillslope) scale and although the predictive maps shown in the figures cannot convey the actual level of spatial details, we uploaded full-resolution maps on an open repository where readers with an interest in our work can download all the outputs produced here. This is meant to ensure full transparency and to share the information in a GIS format that can be used not only for national scale assessments but that can be easily queried also at the regional level and potentially even at the catchment scale.

\section{Conclusions}

The strategy proposed here is currently the most comprehensive example of landslide susceptibility analysis, in a situation where incomplete landslide inventories may affect the model estimates. Is consists in an continuation of the research started with Steger et al. (2016a) and continued in (Steger et al., 2021). Here though, we extend the modeling framework to multiple landslide types and most importantly, we make choices on which sectors to consider inadequate. The decision on which region to consider inadequate relied on a combination of multiple regional intercept, actual technical reports and geomorphological considerations. We maintain that the right approach in similar cases should involve building a model that at least would estimate a series of regression constants per unit of space (here, based on administrative boundaries). The indication provided by multiple intercepts only opens up for further investigation because all it does is to highlight landslide types and regions where the local behavior is less than the national average. We recall here that it is often unknown whether the heterogeneity in the landslide inventory is due to incomplete mapping or to actual differences in the spatial frequency of landslide occurrences. Thus, certain strategies should be considered to discern a real from an artificial effect. We addressed this issue for the Italian landslide inventory by looking into the geomorphological characteristics of the Italian landscape. We assumed that analogous geological and geomorphological clusters (Alvioli et al., 2020) should behave similarly in terms of landsliding. Therefore, by combining the information collected via a multiple-regional-intercept together with the deviation from a consistent landslide behavior measured per cluster, and together with information described in technical reports, we have been able to recognize regions that well aligned with national 
trends and regions that substantially deviated from those.

Specifically, if a cluster would have a certain number of landslides across the whole country and suddenly it reports little to no landslides within a given region, then the indication of a poor local inventory, already provided by the multiple intercept, becomes even more reliable. To this, we then added a series of expert-based checks, which helped confirming or rejecting the incompleteness hypothesis. From an appropriate selection of suitable inventories, we have then fitted a susceptibility model from which thousands of simulations have been generated to characterize the whole Italian territory with a rich probabilistic information. We stress that the same procedure could be largely re-implemented in any study area.

As a result, we proposed for the first time one bias-free landslide susceptibility model for the whole Italian territory and for each landslide type reported in the IFFI inventory.

To promote reproducible results and to allow any reader to access the susceptibility patterns we produced in their raw form, we are sharing the eight mean susceptibility maps and their uncertainty at this link: https://geomorphology.irpi.cnr.it/tools/slope-units.

\section{Acknowledgement}

The research presented in this article is partially supported by King Abdullah University of Science and Technology (KAUST) in Thuwal, Saudi Arabia, Grant URF/1/4338-01-01 and by the Charles University Grant Agency (GAUK; Project No. 337121).

\section{A Summary of simulations}

To simulate over regions with incomplete inventories, we implemented the following procedure. Fitting one susceptibility model per landslide type - solely on the basis of regions that have a complete inventory - allowed us to estimate the posterior distribution of each regression coefficient (global intercept, fixed and random effects; $c f$. Section 3.2). From each posterior distribution, we then extracted 1,000 samples, which we then combined additively in a first step, to estimate the log-odds for regions with a complete inventory. Subsequently, we used the very same 1,000 samples extracted in the previous step, but determined the predictive equation in regions with incomplete landslide inventories. This operation ensured that we have covered the whole Italian territory, and that for each SU, we would have simulated 1,000 log-odds values, which we assumed to be sufficient to describe the mean behavior of landslide occurrences as well as the uncertainty around it. Ultimately, we converted the log-odds into probability values by using the logit link function, Eq. (2), and stored just three parameters out of the 1,000 susceptibility values. These three parameters correspond to the mean, 2.5 and 97.5 percentiles. The difference between the percentiles gives the width of the $95 \%$ credible interval.

It is important to stress a technical requirement one should always consider when simulating over unknown regions while using a random walk (as we did for the mean slope 


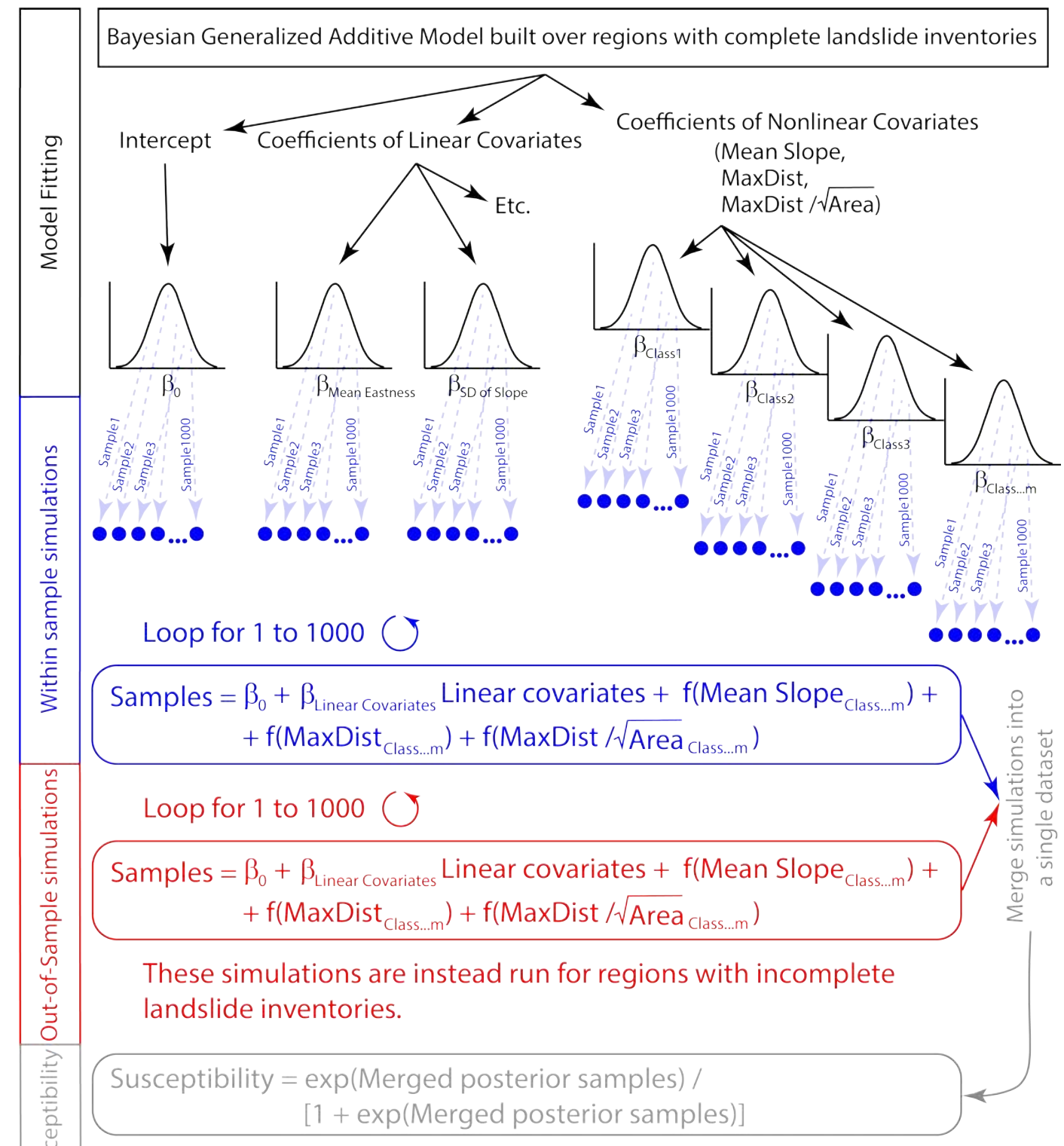

This operation returns 1000 Italian susceptibility maps.

Only the mean, 2.5 and 97.5 percentiles of 1000 susceptibility maps are stored. Three values for each Italian SU and for each landslide type.

Figure 16: Graphical sketch of how we performed the simulations from the regions with a complete inventory to regions with an incomplete one. This figure has been modified from Luo et al. (2021). 
steepness for instance). In such cases, the procedure involves binning the domain of the original covariate into a fixed number of classes on which we then apply the RW1 type model, imposing adjacent class dependence. However, if the domain of the original covariate between the training and the simulated area are very different, then careful choices must be made. To clarify this concept with the reader we can take the mean slope steepness for instance. If the area where we trained the model (with complete inventory) has a range of slope steepness values bewtween 0 and 30 degrees, and the area where we want to simulate for (with incomplete inventories) has a range of slope steepness values bewtween 0 and 60 degrees, then the model would not know what is the effect for values greater than 30 degrees and up to 60 degrees in the simulation phase. In a linear model this issue does not exist as one assumes the effect to be constant irrespective of the value range. However, for random walk models two reasonable choices are available. The first choice, the most conservative, is to fix the same regression coefficient estimated for the 30 degree class up to the 60 degree one. The other option is to consider only the last three or four classes and then use a linear interpolator to extend the regression coefficient estimates up to the desired range. However, this implies a certain degree of expert choice on how many classes to consider for the interpolation; two, three, four or more could all be reasonable choices depending on the specific trend one observes. In our case, we have opted for the first option to contain the amount of subjective influence to our model. We have maintained this choice for the the RW1 type model we used (mean slope steepness, slope unit maximum distance and slope unit elongation/roundness index).

\section{References}

Adams, P. W. and Sidle, R. C. (1987) Soil conditions in three recent landslides in southeast Alaska. Forest Ecology and Management 18(2), 93-102.

Alvioli, M., Guzzetti, F. and Marchesini, I. (2020) Parameter-free delineation of slope units and terrain subdivision of Italy. Geomorphology 358, 107124.

Alvioli, M., Marchesini, I., Reichenbach, P., Rossi, M., Ardizzone, F., Fiorucci, F. and Guzzetti, F. (2016) Automatic delineation of geomorphological slope units with r.slopeunits v1.0 and their optimization for landslide susceptibility modeling. Geoscientific Model Development 9(11), 3975-3991.

Alvioli, M., Mondini, A. C., Fiorucci, F., Cardinali, M. and Marchesini, I. (2018) Topography-driven satellite imagery analysis for landslide mapping. Geomatics, Natural Hazards and Risk 9(1), 544-567.

Alvioli, M., Santangelo, M., Fiorucci, F., Cardinali, M., Marchesini, I., Reichenbach, P., Rossi, M., Guzzetti, F. and Peruccacci, S. (2021) Rockfall susceptibility and networkranked susceptibility along the italian railway. Engineering Geology 293, 106301. 
Amato, G., Eisank, C., Castro-Camilo, D. and Lombardo, L. (2019) Accounting for covariate distributions in slope--unit-based landslide susceptibility models. a case study in the alpine environment. Engineering geology 260, 105237.

Arabameri, A., Rezaei, K., Cerda, A., Lombardo, L. and Rodrigo-Comino, J. (2019) GISbased groundwater potential mapping in Shahroud plain, Iran. A comparison among statistical (bivariate and multivariate), data mining and MCDM approaches. Science of the Total Environment 658, 160-177.

Ardizzone, F., Basile, G., Cardinali, M., Casagli, N., Del Conte, S., Del Ventisette, C., Fiorucci, F., Garfagnoli, F., Gigli, G., Guzzetti, F. et al. (2012) Landslide inventory map for the Briga and the Giampilieri catchments, NE Sicily, Italy. Journal of Maps 8(2), $176-180$.

Arnone, E., Francipane, A., Scarbaci, A., Puglisi, C. and Noto, L. (2016) Effect of raster resolution and polygon-conversion algorithm on landslide susceptibility mapping. Environmental Modelling \& Software 84, 467-481.

Ba, Q., Chen, Y., Deng, S., Yang, J. and Li, H. (2018) A comparison of slope units and grid cells as mapping units for landslide susceptibility assessment. Earth Science Informatics 11(3), 373-388.

Bakka, H., Rue, H., Fuglstad, G.-A., Riebler, A., Bolin, D., Illian, J., Krainski, E., Simpson, D. and Lindgren, F. (2018) Spatial modeling with r-inla: A review. Wiley Interdisciplinary Reviews: Computational Statistics 10(6), e1443.

Bartolini, C. (2010) Outline of Italy's Geomorphology. Journal of the Virtual Explorer 36.

Bianchini, S., Cigna, F., Moretti, S. and Casagli, N. (2013) Monitoring landslide-induced displacements with TerraSAR-X persistent scatterer interferometry (PSI): Gimigliano case study in Calabria region (Italy). International Journal of Geosciences 4(10), 1467.

Bini, C. (2013) Geology and Geomorphology. In The Soils of Italy, eds E. A. Costantini and C. Dazzi, World Soils Book Series, pp. 39-56. Dordrecht: Springer Netherlands. ISBN 978-94-007-5642-7.

Böhner, J. and Selige, T. (2006) Spatial prediction of soil attributes using terrain analysis and climate regionalisation. Gottinger Geographische Abhandlungen 115, 13-28.

Bornaetxea, T. and Marchesini, I. (2021) r.survey: a tool for calculating visibility of variablesize objects based on orientation. International Journal of Geographical Information Science pp. 1-24.

Bornaetxea, T., Rossi, M., Marchesini, I. and Alvioli, M. (2018) Effective surveyed area and its role in statistical landslide susceptibility assessments. Natural Hazards and Earth System Sciences 18(9), 2455-2469. 
Bosellini, A. (2017) Outline of the Geology of Italy. In Landscapes and Landforms of Italy, eds M. Soldati and M. Marchetti, World Geomorphological Landscapes, pp. 21-27. Cham: Springer International Publishing. ISBN 978-3-319-26194-2.

Van den Bout, B., Lombardo, L., Chiyang, M., van Westen, C. and Jetten, V. (2021) Physically-based catchment-scale prediction of slope failure volume and geometry. Engineering Geology 284, 105942.

Bout, B., Lombardo, L., van Westen, C. and Jetten, V. (2018) Integration of two-phase solid fluid equations in a catchment model for flashfloods, debris flows and shallow slope failures. Environmental Modelling \& Software 105, 1-16.

Budimir, M., Atkinson, P. and Lewis, H. (2015) A systematic review of landslide probability mapping using logistic regression. Landslides 12(3), 419-436.

Cama, M., Lombardo, L., Conoscenti, C., Agnesi, V. and Rotigliano, E. (2015) Predicting storm-triggered debris flow events: application to the 2009 Ionian Peloritan disaster (Sicily, Italy). Nat Hazards Earth Syst Sci 15(8), 1785-1806.

Cama, M., Lombardo, L., Conoscenti, C. and Rotigliano, E. (2017) Improving transferability strategies for debris flow susceptibility assessment: Application to the Saponara and Itala catchments (Messina, Italy). Geomorphology 288, 52-65.

Castro Camilo, D., Lombardo, L., Mai, P., Dou, J. and Huser, R. (2017) Handling high predictor dimensionality in slope-unit-based landslide susceptibility models through LASSOpenalized Generalized Linear Model. Environmental Modelling and Software 97, 145-156.

Ciampalini, A., Raspini, F., Bianchini, S., Frodella, W., Bardi, F., Lagomarsino, D., Di Traglia, F., Moretti, S., Proietti, C., Pagliara, P. et al. (2015) Remote sensing as tool for development of landslide databases: the case of the Messina province (Italy) geodatabase. Geomorphology 249, 103-118.

Colombo, A., Lanteri, L., Ramasco, M. and Troisi, C. (2005) Systematic gis-based landslide inventory as the first step for effective landslide-hazard management. Landslides 2(4), 291-301.

Cowie, P. A., Phillips, R. J., Roberts, G. P., McCaffrey, K., Zijerveld, L. J. J., Gregory, L. C., Faure Walker, J., Wedmore, L. N. J., Dunai, T. J., Binnie, S. A., Freeman, S. P. H. T., Wilcken, K., Shanks, R. P., Huismans, R. S., Papanikolaou, I., Michetti, A. M. and Wilkinson, M. (2017) Orogen-scale uplift in the central Italian Apennines drives episodic behaviour of earthquake faults. Scientific Reports 7(1), 44858.

Das, I., Stein, A., Kerle, N. and Dadhwal, V. K. (2012) Landslide susceptibility mapping along road corridors in the Indian Himalayas using Bayesian logistic regression models. Geomorphology 179, 116-125. 
Devoli, G., Kleivane, I., Sund, M., Orthe, N.-K., Ekker, R., Johnsen, E. and Colleuille, H. (2015) Landslide early warning system and web tools for real-time scenarios and for distribution of warning messages in norway. In Engineering Geology for Society and Territory - Volume 2, eds G. Lollino, D. Giordan, G. B. Crosta, J. Corominas, R. Azzam, J. Wasowski and N. Sciarra, pp. 625-629. Cham: Springer International Publishing. ISBN 978-3-319-09057-3.

Doménech, G., Alvioli, M. and Corominas, J. (2020) Preparing first-time slope failures hazard maps: from pixel-based to slope unit-based. Landslides 17, 249-265.

Drgu, L. and Eisank, C. (2012) Automated object-based classification of topography from SRTM data. Geomorphology 141-142, 21-33.

Erener, A. and Düzgün, H. S. B. (2012) Landslide susceptibility assessment: what are the effects of mapping unit and mapping method? Environmental Earth Sciences 66(3), 859-877.

Fan, X., Scaringi, G., Korup, O., West, A. J., van Westen, C. J., Tanyas, H., Hovius, N., Hales, T. C., Jibson, R. W., Allstadt, K. E. et al. (2019) Earthquake-induced chains of geologic hazards: Patterns, mechanisms, and impacts. Reviews of geophysics 57(2), $421-503$.

Forman, R. T. and Godron, M. (1986) Landscape ecology. New York 4, 22-28.

Frattini, P., Crosta, G. and Carrara, A. (2010) Techniques for evaluating the performance of landslide susceptibility models. Engineering Geology 111(1), 62-72.

Fredi, P. and Lupia Palmieri, E. (2017) Morphological Regions of Italy, pp. 39-74. Cham: Springer International Publishing. ISBN 978-3-319-26194-2.

Galli, M., Ardizzone, F., Cardinali, M., Guzzetti, F. and Reichenbach, P. (2008) Comparing landslide inventory maps. Geomorphology 94(3), 268-289. GIS technology and models for assessing landslide hazard and risk.

Garson, G. D. (2013) Fundamentals of hierarchical linear and multilevel modeling. Hierarchical linear modeling: Guide and applications pp. 3-25.

Goetz, J., Kohrs, R., Parra Hormazábal, E., Bustos Morales, M., Belén Araneda Riquelme, M., Henríquez, C. and Brenning, A. (2021) Optimizing and validating the Gravitational Process Path model for regional debris-flow runout modelling. Natural Hazards and Earth System Sciences Discussions pp. 1-30.

Goetz, J. N., Guthrie, R. H. and Brenning, A. (2011) Integrating physical and empirical landslide susceptibility models using generalized additive models. Geomorphology 129(34), 376-386. 
Guerricchio, A., Doglioni, A., Fortunato, G., Galeandro, A., Guglielmo, E., Versace, P. and Simeone, V. (2012) Landslide hazard connected to deep seated gravitational slope deformations and prolonged rainfall: Maierato landslide case history. Società Geologica Italiana 21, 574-576.

Guzzetti, F., Mondini, A. C., Cardinali, M., Fiorucci, F., Santangelo, M. and Chang, K.-T. (2012) Landslide inventory maps: New tools for an old problem. Earth-Science Reviews 112(1-2), 42-66.

Guzzetti, F. and Reichenbach, P. (1994) Towards a definition of topographic divisions for Italy. Geomorphology 11(1), 57-74.

Heerdegen, R. G. and Beran, M. A. (1982) Quantifying source areas through land surface curvature and shape. Journal of Hydrology 57(3-4), 359-373.

Hengl, T., de Jesus, J. M., Heuvelink, G. B., Gonzalez, M. R., Kilibarda, M., Blagotić, A., Shangguan, W., Wright, M. N., Geng, X., Bauer-Marschallinger, B. et al. (2017) SoilGrids250m: Global gridded soil information based on machine learning. PLoS one 12(2), e0169748.

Hervás, J. and Bobrowsky, P. (2009) Mapping: inventories, susceptibility, hazard and risk. In Landslides-disaster risk reduction, pp. 321-349. Springer.

Hölbling, D., Füreder, P., Antolini, F., Cigna, F., Casagli, N. and Lang, S. (2012) A semiautomated object-based approach for landslide detection validated by persistent scatterer interferometry measures and landslide inventories. Remote Sensing 4(5), 1310-1336.

Hosmer, D. W. and Lemeshow, S. (2000) Applied Logistic Regression. Second edition. New York: Wiley.

Huang, F., Yin, K., Huang, J., Gui, L. and Wang, P. (2017) Landslide susceptibility mapping based on self-organizing-map network and extreme learning machine. Engineering Geology 223, 11-22.

Hungr, O., Leroueil, S. and Picarelli, L. (2014) The Varnes classification of landslide types, an update. Landslides 11(2), 167-194.

Iadanza, C., Trigila, A. and Napolitano, F. (2016) Identification and characterization of rainfall events responsible for triggering of debris flows and shallow landslides. Journal of Hydrology 541, 230-245.

Jacobs, L., Kervyn, M., Reichenbach, P., Rossi, M., Marchesini, I., Alvioli, M. and Dewitte, O. (2020) Regional susceptibility assessments with heterogeneous landslide information: Slope unit--vs. pixel-based approach. Geomorphology 356, 107084. 
Kirschbaum, D., Stanley, T. and Zhou, Y. (2015) Spatial and temporal analysis of a global landslide catalog. Geomorphology 249, 4-15.

Knevels, R., Petschko, H., Proske, H., Leopold, P., Maraun, D. and Brenning, A. (2020) Event-based landslide modeling in the Styrian Basin, Austria: accounting for time-varying rainfall and land cover. Geosciences 10(6), 217.

Korup, O. (2021) Bayesian geomorphology. Earth Surface Processes and Landforms 46(1), $151-172$.

Lima, P., Steger, S. and Glade, T. (2021) Counteracting flawed landslide data in statistically based landslide susceptibility modelling for very large areas: a national-scale assessment for Austria. Landslides pp. 1-16.

Lima, P., Steger, S., Glade, T., Tilch, N., Schwarz, L. and Kociu, A. (2017) Landslide susceptibility mapping at national scale: a first attempt for austria. In Workshop on World Landslide Forum, pp. 943-951.

Lin, Q., Lima, P., Steger, S., Glade, T., Jiang, T., Zhang, J., Liu, T. and Wang, Y. (2021) National-scale data-driven rainfall induced landslide susceptibility mapping for China by accounting for incomplete landslide data. Geoscience Frontiers p. 101248.

Lindgren, F. and Rue, H. (2015) Bayesian spatial modelling with R-INLA. Journal of Statistical Software 63(19), 1-25.

Loche, M., Scaringi, G., Blaht, J., Melis, M. T., Funedda, A., Da Pelo, S., Erb, I., Deiana, G., Meloni, M. A. and Cocco, F. (2021) An infrared thermography approach to evaluate the strength of a rock cliff. Remote Sensing 13(7).

Loche, M., Scaringi, G., Yunus, A. P., Catani, F., Tanyaş, H., Frodella, W., Fan, X. and Lombardo, L. (2022) Surface temperature controls the pattern of post-earthquake landslide activity. Scientific Reports 12(1).

Lombardo, L., Cama, M., Conoscenti, C., Märker, M. and Rotigliano, E. (2015) Binary logistic regression versus stochastic gradient boosted decision trees in assessing landslide susceptibility for multiple-occurring landslide events: application to the 2009 storm event in Messina (Sicily, southern Italy). Natural Hazards 79(3), 1621-1648.

Lombardo, L., Fubelli, G., Amato, G. and Bonasera, M. (2016) Presence-only approach to assess landslide triggering-thickness susceptibility: a test for the Mili catchment (northeastern Sicily, Italy). Natural Hazards 84(1), 565-588.

Lombardo, L. and Mai, P. M. (2018) Presenting logistic regression-based landslide susceptibility results. Engineering geology 244, 14-24. 
Lombardo, L., Opitz, T. and Huser, R. (2018a) Point process-based modeling of multiple debris flow landslides using INLA: an application to the 2009 Messina disaster. Stochastic Environmental Research and Risk Assessment 32(7), 2179-2198.

Lombardo, L., Opitz, T. and Huser, R. (2019) Numerical Recipes for Landslide Spatial Prediction Using R-INLA: A Step-by-Step Tutorial. Spatial Modeling in GIS and R for Earth and Environmental Sciences p. 55.

Lombardo, L., Saia, S., Schillaci, C., Mai, P. M. and Huser, R. (2018b) Modeling soil organic carbon with Quantile Regression: Dissecting predictors' effects on carbon stocks. Geoderma 318, 148-159.

Lombardo, L. and Tanyas, H. (2020) Chrono-validation of near-real-time landslide susceptibility models via plug-in statistical simulations. Engineering Geology 278, 105818.

Lombardo, L. and Tanyas, H. (2021) From scenario-based seismic hazard to scenario-based landslide hazard: fast-forwarding to the future via statistical simulations. Stochastic Environmental Research and Risk Assessment pp. 1-14.

Lombardo, L., Tanyas, H. and Nicu, I. C. (2020) Spatial modeling of multi-hazard threat to cultural heritage sites. Engineering Geology 277, 105776.

Luo, L., Lombardo, L., van Westen, C., Pei, X. and Huang, R. (2021) From scenario-based seismic hazard to scenario-based landslide hazard: rewinding to the past via statistical simulations. Stochastic environmental research and risk assessment pp. 1-22.

Marchesini, I., Ardizzone, F., Alvioli, M., Rossi, M. and Guzzetti, F. (2014) Non-susceptible landslide areas in italy and in the mediterranean region. Natural Hazards and Earth System Sciences 14(8), 2215-2231.

Nefeslioglu, H. A., Gokceoglu, C. and Sonmez, H. (2008) An assessment on the use of logistic regression and artificial neural networks with different sampling strategies for the preparation of landslide susceptibility maps. Engineering Geology 97(3-4), 171-191.

Opitz, T., Huser, R., Bakka, H. and Rue, H. (2018) INLA goes extreme: Bayesian tail regression for the estimation of high spatio-temporal quantiles. Extremes 21(3), 441-462.

Petschko, H., Bell, R., Brenning, A. and Glade, T. (2012) Landslide susceptibility modeling with generalized additive models-facing the heterogeneity of large regions. Landslides and Engineered Slopes, Protecting Society through Improved Understanding 1, 769-777.

Petschko, H., Brenning, A., Bell, R., Goetz, J. and Glade, T. (2014) Assessing the quality of landslide susceptibility maps-case study Lower Austria. Natural Hazards and Earth System Sciences 14(1), 95-118. 
Pimont, F., Fargeon, H., Opitz, T., Ruffault, J., Barbero, R., Martin-StPaul, N., Rigolot, E., Rivière, M. and Dupuy, J.-L. (2021) Prediction of regional wildfire activity in the probabilistic Bayesian framework of Firelihood. Ecological applications 31(5), e02316.

Pokharel, B., Alvioli, M. and Lim, S. (2021) Assessment of earthquake-induced landslide inventories and susceptibility maps using slope unit-based logistic regression and geospatial statistics. Scientific Reports 11(2133), 1-15.

Rahmati, O., Kornejady, A., Samadi, M., Deo, R. C., Conoscenti, C., Lombardo, L., Dayal, K., Taghizadeh-Mehrjardi, R., Pourghasemi, H. R., Kumar, S. et al. (2019) Pmt: New analytical framework for automated evaluation of geo-environmental modelling approaches. Science of the total environment 664, 296-311.

Reichenbach, P., Rossi, M., Malamud, B., Mihir, M. and Guzzetti, F. (2018) A review of statistically-based landslide susceptibility models. Earth-Science Reviews 180, 60-91.

Rossi, M., Guzzetti, F., Reichenbach, P., Mondini, A. C. and Peruccacci, S. (2010) Optimal landslide susceptibility zonation based on multiple forecasts. Geomorphology 114(3), 129142.

Rossi, M., Guzzetti, F., Salvati, P., Donnini, M., Napolitano, E. and Bianchi, C. (2019) A predictive model of societal landslide risk in Italy. Earth-Science Reviews 196, 102849.

Rue, H. and Held, L. (2005) Gaussian Markov random fields: theory and applications. Chapman and Hall/CRC.

Rue, H., Martino, S. and Chopin, N. (2009) Approximate Bayesian inference for latent Gaussian models by using integrated nested Laplace approximations. Journal of the Royal Statistical Society: Series B 71(2), 319-392.

Sala, G., Lanfranconi, C., Frattini, P., Rusconi, G. and Crosta, G. B. (2021) Cost-sensitive rainfall thresholds for shallow landslides. Landslides pp. 1-14.

Scaringi, G. and Loche, M. (2022) A thermo-hydro-mechanical approach to soil slope stability under climate change. Geomorphology p. 108108.

Schlögel, R., Marchesini, I., Alvioli, M., Reichenbach, P., Rossi, M. and Malet, J.-P. (2018) Optimizing landslide susceptibility zonation: Effects of dem spatial resolution and slope unit delineation on logistic regression models. Geomorphology 301, 10-20.

Segoni, S., Lagomarsino, D., Fanti, R., Moretti, S. and Casagli, N. (2015) Integration of rainfall thresholds and susceptibility maps in the Emilia Romagna (Italy) regional-scale landslide warning system. Landslides 12(4), 773-785. 
Segui, C., Rattez, H. and Veveakis, M. (2020) On the stability of deep-seated landslides. the cases of vaiont (italy) and shuping (three gorges dam, china). Journal of Geophysical Research: Earth Surface 125(7), e2019JF005203.

Simpson, D., Rue, H., Riebler, A., Martins, T. G. and Sørbye, S. H. (2017) Penalising model component complexity: A principled, practical approach to constructing priors. Statistical science 32(1), 1-28.

Soldati, M. and Marchetti, M. (2017) Landscapes and landforms of Italy. Springer.

Steger, S., Brenning, A., Bell, R. and Glade, T. (2016a) The propagation of inventorybased positional errors into statistical landslide susceptibility models. Natural Hazards and Earth System Sciences 16(12), 2729-2745.

Steger, S., Brenning, A., Bell, R., Petschko, H. and Glade, T. (2016b) Exploring discrepancies between quantitative validation results and the geomorphic plausibility of statistical landslide susceptibility maps. Geomorphology 262, 8-23.

Steger, S., Mair, V., Kofler, C., Pittore, M., Zebisch, M. and Schneiderbauer, S. (2021) Correlation does not imply geomorphic causation in data-driven landslide susceptibility modelling-Benefits of exploring landslide data collection effects. Science of the total environment 776, 145935.

Tanyaş, H., van Westen, C., Allstadt, K., Nowicki, A. J. M., Görüm, T., Jibson, R., Godt, J., Sato, H., Schmitt, R., Marc, O. and Hovius, N. (2017) Presentation and Analysis of a Worldwide Database of Earthquake-Induced Landslide Inventories. Journal of Geophysical Research: Earth Surface 122(10), 1991-2015.

Tanyaş, H., Görüm, T., Kirschbaum, D. and Lombardo, L. (2022) Could road constructions be more hazardous than an earthquake in terms of mass movement? Natural Hazards pp. $1-25$.

Tanyaş, H., Hill, K., Mahoney, L., Fadel, I. and Lombardo, L. (2021) The world's secondlargest, recorded landslide event: Lessons learnt from the landslides triggered during and after the $2018 \mathrm{Mw} 7.5$ Papua New Guinea earthquake. Engineering Geology p. 106504.

Tanyaş, H. and Lombardo, L. (2020) Completeness Index for Earthquake-Induced Landslide Inventories. Engineering geology 264, 105331.

Tanyas, H. and Lombardo, L. (2020) Completeness index for earthquake-induced landslide inventories. Engineering geology 264, 105331.

Tanyaş, H., Rossi, M., Alvioli, M., van Westen, C. J. and Marchesini, I. (2019a) A global slope unit-based method for the near real-time prediction of earthquake-induced landslides. Geomorphology 327, 126-146. 
Tanyaş, H., van Westen, C. J., Persello, C. and Alvioli, M. (2019b) Rapid prediction of the magnitude scale of landslide events triggered by an earthquake. Landslides 16(4), 661-676.

Titti, G., van Westen, C., Borgatti, L., Pasuto, A. and Lombardo, L. (2021) When Enough Is Really Enough? On the Minimum Number of Landslides to Build Reliable Susceptibility Models. Geosciences 11(11), 469.

Trigila, A., Iadanza, C. and Guerrieri, L. (2007) The IFFI project (Italian landslide inventory): Methodology and results. Guidelines for Mapping Areas at Risk of Landslides in Europe 23, 15.

Trigila, A., Iadanza, C. and Spizzichino, D. (2010) Quality assessment of the italian landslide inventory using gis processing. Landslides 7(4), 455-470.

Van Den Eeckhaut, M., Hervás, J., Jaedicke, C., Malet, J.-P., Montanarella, L. and Nadim, F. (2012) Statistical modelling of Europe-wide landslide susceptibility using limited landslide inventory data. Landslides 9(3), 357-369.

Varnes, D. J. (1978) Slope movement types and processes. Special report 176, 11-33.

Yesilnacar, E. and Topal, T. (2005) Landslide susceptibility mapping: a comparison of logistic regression and neural networks methods in a medium scale study, Hendek region (Turkey). Engineering Geology 79(3-4), 251-266.

Zevenbergen, L. W. and Thorne, C. R. (1987) Quantitative analysis of land surface topography. Earth surface processes and landforms 12(1), 47-56. 